\title{
Optimal Exponential Synchronization of Chaotic Systems with Multiple Time Delays via Fuzzy Control
}

\author{
Feng-Hsiag Hsiao \\ Department of Electrical Engineering, National University of Tainan, No. 33, Section 2, Shu Lin Street, \\ Tainan 700, Taiwan
}

Correspondence should be addressed to Feng-Hsiag Hsiao; fhhsiao@mail.nutn.edu.tw

Received 15 January 2013; Revised 26 April 2013; Accepted 28 April 2013

Academic Editor: Ryan Loxton

Copyright (C) 2013 Feng-Hsiag Hsiao. This is an open access article distributed under the Creative Commons Attribution License, which permits unrestricted use, distribution, and reproduction in any medium, provided the original work is properly cited.

This study presents an effective approach to realize the optimal $H^{\infty}$ exponential synchronization of multiple time-delay chaotic (MTDC) systems. First, a neural network (NN) model is employed to approximate the MTDC system. Then, a linear differential inclusion (LDI) state-space representation is established for the dynamics of the NN model. Based on this LDI state-space representation, this study proposes a delay-dependent exponential stability criterion of the error system derived in terms of Lyapunov's direct method to ensure that the trajectories of the slave system can approach those of the master system. Subsequently, the stability condition of this criterion is reformulated into a linear matrix inequality (LMI). Based on the LMI, a fuzzy controller is synthesized not only to realize the exponential synchronization but also to achieve the optimal $H^{\infty}$ performance by minimizing the disturbance attenuation level. Finally, a numerical example with simulations is provided to illustrate the concepts discussed throughout this work.

\section{Introduction}

In practice, due to information transmission, time delays naturally exist in many systems. The existence of time delay is frequently a source of instability and is encountered in various engineering systems [1-5]. Consequently, the problem of stability analysis in time-delay systems remains a major focus of researchers wishing to inspect the properties of such systems.

Since chaotic phenomenon in time-delay systems was first found by Mackey and Glass [6], there has been increasing interest in time-delay chaotic systems. Chaos is a wellknown nonlinear phenomenon, and it is irregular, seemingly random and extremely sensitive to initial conditions [7]. Based on these properties, chaos has received a great deal of interest among scientists from various research fields [812]. One of its research fields for communication, chaotic synchronization, has been investigated extensively.
The chaotic synchronization proposed by Pecora and Carroll in 1990 [13] is intended to control one chaotic system to follow another. Since the introduction of this concept, various synchronization approaches, such as nonlinear feedback control [14] and adaptive control [15], have been widely developed in the past two decades. Chaos synchronization can be applied in the vast areas of physics and engineering science, especially in secure communication [16]. Therefore, chaotic synchronization has become a popular study [14-23].

In real physical systems, some noises or disturbances always exist that may cause instability and thereby destroy the synchronization performance. Hence, how to reduce the effect of external disturbances in synchronization process for chaotic systems is an important issue $[24,25]$. The $H^{\infty}$ control has been conferred for synchronization in chaotic systems over the last few years [24-28]. And the $H^{\infty}$ synchronization problem was also investigated extensively for timedelay chaotic systems (see, e.g., [25, 29-31]). Accordingly, 
the objective of this study is to realize the exponential synchronization of multiple time-delay chaotic (MTDC) systems, and at the same time the effect of external disturbance on control performance is attenuated to a minimum level.

Neural-network- (NN-) based modeling has become an active research field in the past few years due to its unique merits in solving complex nonlinear system identification and control problems [32-37]. Over the past decade, fuzzy control has rapidly developed in both the academic and industrial communities and there have been many successful applications. Despite the successes of fuzzy control, it has become evident that many basic problems remain to be solved. Stability analysis and systematic design are certainly among the most important issues for fuzzy control systems. Lately, there have been significant research efforts devoted to these issues (see [38-41]). However, all of them neglect the modeling errors between nonlinear systems and fuzzy models. The existence of modeling errors may be a potential source of instability for control designs based on the assumption that the fuzzy model exactly matches the nonlinear plant [42]. Recently, Kiriakidis [42], Chen et al. [43, 44], and Cao et al. $[45,46]$ proposed novel approaches to overcome the influence of modeling errors in the field of model-based fuzzy control for nonlinear systems.

Consequently, an effective method is proposed via neural-network- (NN-) based technique to realize the optimal $H^{\infty}$ exponential synchronization of multiple time-delay chaotic (MTDC) systems in this study. Based on the above, the trajectories of slave systems can approach those of master systems and the effect of external disturbance on control performance is attenuated to a minimum level.

This study is organized as follows. The system description is arranged in Section 2. In Section 3, a robustness design of fuzzy controllers is proposed to realize the optimal $H^{\infty}$ exponential synchronization. The design algorithm is shown in Section 4. In Section 5, the effectiveness of the proposed approach is illustrated by a numerical example. Finally, the conclusions are drawn in Section 6.

\section{System Description}

Consider a pair of multiple time-delay chaotic (MTDC) systems in master-slave configuration. The dynamics of the master system $\left(N_{m}\right)$ and slave system $\left(N_{s}\right)$ are described as follows:

$$
\begin{gathered}
N_{m}: \dot{X}(t)=f(X(t))+\sum_{k=1}^{m} H_{k}\left(X\left(t-\tau_{k}\right)\right), \\
N_{s}: \widehat{\dot{X}}(t)=f(\widehat{X}(t))+\sum_{k=1}^{m} H_{k}\left(\widehat{X}\left(t-\tau_{k}\right)\right)+B U(t)+\partial(t),
\end{gathered}
$$

where $f(\cdot)$ and $H_{k}(\cdot)$ are the nonlinear vector-valued functions, $\partial(t)$ denotes the external disturbance, $\tau_{k}(k=$ $1,2, \ldots, m)$ are the time delays, and $U(t)$ is the control input. Moreover, $X(t)$ and $\widehat{X}(t)$ are the state vectors of $N_{m}$ and $N_{s}$, respectively.

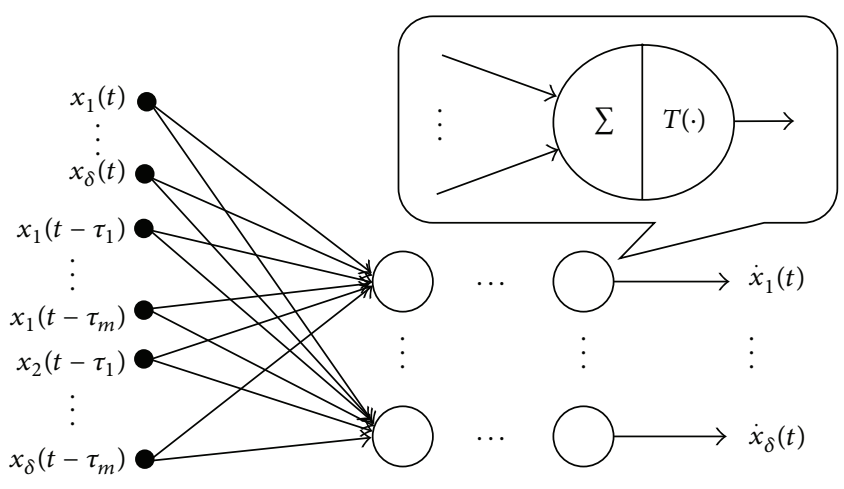

FIGURE 1: An NN model.

In this section, a neural network (NN) model is first established to approximate the MTDC system. The dynamics of the NN model are then converted into a linear differential inclusion (LDI) state-space representation. Finally, on the basis of the LDI state-space representation, a fuzzy controller is synthesized to realize the synchronization of MTDC systems.

2.1. Neural Network (NN) Model. The MTDC system can be approximated by an NN model, as shown in Figure 1, that has $S$ layers with $J^{\sigma}(\sigma=1,2, \ldots, S)$ neurons for each layer, in which $x_{1}(t) \sim x_{\delta}(t)$ are the state variables and $x_{1}\left(t-\tau_{1}\right) \sim$ $x_{1}\left(t-\tau_{m}\right), x_{2}\left(t-\tau_{1}\right) \sim x_{\delta}\left(t-\tau_{m}\right)$ are the state variables with delays.

To distinguish among these layers, the superscripts are used for identifying the layers. Specifically, we append the number of the layer as a superscript to the names for each of these variables. Thus, the weight matrix for the $\sigma$ th layer is written as $W^{\sigma}$. Moreover, it is assumed that $v_{\varsigma}^{\sigma}(t)(\varsigma=$ $\left.1,2, \ldots, J^{\sigma} ; \sigma=1,2, \ldots, S\right)$ is the net input and $T\left(v_{\varsigma}^{\sigma}(t)\right)$ is the transfer function of the neuron. Subsequently, the transfer function vector of the $\sigma$ th layer is defined as

$$
\begin{array}{r}
\Psi^{\sigma}\left(v_{\varsigma}^{\sigma}(t)\right) \equiv\left[T\left(v_{1}^{\sigma}(t)\right) T\left(v_{2}^{\sigma}(t)\right) \cdots T\left(v_{J^{\sigma}}^{\sigma}(t)\right)\right]^{T}, \\
\sigma=1,2, \ldots, S,
\end{array}
$$

where $T\left(v_{\varsigma}^{\sigma}(t)\right)\left(\varsigma=1,2, \ldots, J^{\sigma}\right)$ is the transfer function of the $\varsigma$ th neuron. The final output of NN model can then be inferred as follows:

$$
\begin{aligned}
\dot{X}(t)=\Psi^{S}\left(W^{S} \Psi^{S-1}\right. & \left(W^{S-1} \Psi^{S-2}\right. \\
& \left.\times\left(\cdots \Psi^{2}\left(W^{2} \Psi^{1}\left(W^{1} \Lambda(t)\right)\right) \cdots\right)\right),
\end{aligned}
$$


where

$$
\Lambda^{T}(t)=\left[\begin{array}{ll}
X^{T}(t) & X^{T}\left(t-\tau_{k}\right)
\end{array}\right]
$$

with

$$
\begin{gathered}
X(t)=\left[x_{1}(t) x_{2}(t) \cdots x_{\delta}(t)\right]^{T}, \\
X\left(t-\tau_{k}\right)=\left[x_{1}\left(t-\tau_{1}\right) \cdots x_{1}\left(t-\tau_{m}\right) x_{2}\left(t-\tau_{1}\right)\right. \\
\left.\quad \cdots x_{\delta}\left(t-\tau_{m}\right)\right]^{T} \quad \text { for } k=1,2, \ldots, m .
\end{gathered}
$$

2.2. Linear Differential Inclusion (LDI). To deal with the synchronization problem of MTDC systems, this study establishes the following LDI state-space representation for the dynamics of the NN model, described as [47, 48]:

$$
\begin{gathered}
\dot{O}(t)=A(a(t)) O(t), \\
A(a(t))=\sum_{i=1}^{\phi} h_{i}(a(t)) \widetilde{A}_{i},
\end{gathered}
$$

where $\phi$ is a positive integer, $a(t)$ is a vector signifying the dependence of $h_{i}(\cdot)$ on its elements, $\widetilde{A}_{i}(i=1,2, \ldots, \phi)$ are constant matrices, and $O(t)=\left[\begin{array}{llll}o_{1}(t) & o_{2}(t) & \cdots & o_{\aleph}(t)\end{array}\right]^{T}$. Furthermore, it is assumed that $h_{i}(a(t)) \geq 0$ and $\sum_{i=1}^{\phi} h_{i}(a(t))=1$. Based on the properties of LDI, without loss of generality, we can use $h_{i}(t)$ instead of $h_{i}(a(t))$. The following procedure represents the dynamics of the NN model (4) by LDI state-space representation [47].

To begin with, notice that the output $T\left(v_{c}^{\sigma}(t)\right)$ satisfies

$$
\begin{array}{ll}
g_{\zeta 0}^{\sigma} v_{\zeta}^{\sigma}(t) \leq T\left(v_{\varsigma}^{\sigma}(t)\right) \leq g_{\zeta 1}^{\sigma} v_{\varsigma}^{\sigma}(t), & v_{\varsigma}^{\sigma}(t) \geq 0, \\
g_{\zeta 1}^{\sigma} v_{\zeta}^{\sigma}(t) \leq T\left(v_{\varsigma}^{\sigma}(t)\right) \leq g_{\zeta 0}^{\sigma} v_{\varsigma}^{\sigma}(t), & v_{\varsigma}^{\sigma}(t)<0,
\end{array}
$$

where $g_{\varsigma 0}^{\sigma}$ and $g_{\varsigma 1}^{\sigma}$ denote the minimum and the maximum of the derivative of $T\left(v_{\varsigma}^{\sigma}(t)\right)$, respectively, and are given in the following:

$$
g_{\varsigma \varphi}^{\sigma}= \begin{cases}\min _{v} \frac{d T\left(v_{\varsigma}^{\sigma}(t)\right)}{d v_{\varsigma}^{\sigma}(t)} & \text { when } \varphi=0, \\ \max _{\nu} \frac{d T\left(v_{\varsigma}^{\sigma}(t)\right)}{d v_{\varsigma}^{\sigma}(t)} & \text { when } \varphi=1 .\end{cases}
$$

Subsequently, the min-max matrix $G^{\sigma}$ of the $\sigma$ th layer is defined as follows:

$$
G^{\sigma} \equiv \operatorname{diag}\left[g_{\varsigma \varphi_{\varsigma}}^{\sigma}\right]=\left[\begin{array}{ccccc}
g_{1 \varphi_{1}}^{\sigma} & 0 & 0 & \cdots & 0 \\
0 & g_{2 \varphi_{2}}^{\sigma} & 0 & \ddots & 0 \\
0 & 0 & g_{3 \varphi_{3}}^{\sigma} & 0 & \vdots \\
\vdots & \ddots & 0 & \ddots & 0 \\
0 & 0 & \cdots & 0 & g_{J^{\sigma} \varphi_{J}}^{\sigma}
\end{array}\right] .
$$

Moreover, based on the interpolation method, the transfer function $T\left(v_{\varsigma}^{\sigma}(t)\right)$ can be represented as follows [47]:

$$
\begin{aligned}
T\left(v_{\varsigma}^{\sigma}(t)\right) & =\left(h_{\varsigma 0}^{\sigma}(t) g_{\varsigma 0}^{\sigma}+h_{\varsigma 1}^{\sigma}(t) g_{\varsigma 1}^{\sigma}\right) v_{\varsigma}^{\sigma}(t) \\
& =\left(\sum_{\varphi=0}^{1} h_{\varsigma \varphi}^{\sigma}(t) g_{\varsigma \varphi}^{\sigma}\right) v_{\varsigma}^{\sigma}(t)
\end{aligned}
$$

where the interpolation coefficients $h_{\varsigma \varphi}^{\sigma}(t) \in[0,1]$ and $\sum_{\varphi=0}^{1} h_{\zeta \varphi}^{\sigma}(t)=1$. Equations (3) and (11) show that

$$
\begin{aligned}
& \Psi^{\sigma}\left(v_{\varsigma}^{\sigma}(t)\right) \equiv\left[\begin{array}{lllll}
T\left(v_{1}^{\sigma}(t)\right) & T\left(v_{2}^{\sigma}(t)\right) & \cdots & T\left(v_{J^{\sigma}}^{\sigma}(t)\right)
\end{array}\right]^{T} \\
& =\left[\left(\sum_{\varphi_{1}=0}^{1} h_{1 \varphi_{1}}^{\sigma}(t) g_{1 \varphi_{1}}^{\sigma}\right) v_{1}^{\sigma}(t)\left(\sum_{\varphi_{2}=0}^{1} h_{2 \varphi_{2}}^{\sigma}(t) g_{2 \varphi_{2}}^{\sigma}\right)\right. \\
& \left.\times v_{2}^{\sigma}(t) \cdots\left(\sum_{\varphi_{J}=0}^{1} h_{J^{\sigma} \varphi_{J}}^{\sigma}(t) g_{J^{\sigma} \varphi_{J}}^{\sigma}\right) v_{J^{\sigma}}^{\sigma}(t)\right]^{T} .
\end{aligned}
$$

Therefore, the final output of the NN model (4) can be reformulated as follows:

$$
\begin{gathered}
\dot{X}(t)=\sum_{p=0}^{1} h_{\varsigma p}^{S}(t) G^{S} \\
\times\left(W ^ { S } \ldots \left[\sum_{n=0}^{1} h_{\varsigma n}^{2}(t) G^{2}\right.\right. \\
\times\left(W ^ { 2 } \left[\sum_{b=0}^{1} h_{\varsigma b}^{1}(t) G^{1}\right.\right. \\
\left.\left.\left.\left.\left.\times\left(W^{1} \Lambda(t)\right)\right]\right)\right] \cdots\right]\right) \\
=\sum_{p=0}^{1} \ldots \sum_{n=0}^{1} \sum_{b=0}^{1} h_{\varsigma p}^{S}(t) \cdots h_{\varsigma n}^{2}(t) h_{\varsigma b}^{1}(t) G^{S} W^{S} \\
\cdots G^{2} W^{2} G^{1} W^{1} \Lambda(t) \\
=\sum_{\Omega} h_{\varsigma \Omega}^{\sigma}(t) C_{\Omega}^{\sigma} \Lambda(t),
\end{gathered}
$$


where

$$
\begin{gathered}
\sum_{b=0}^{1} h_{c b}^{1}(t) \equiv \sum_{b_{1}=0}^{1} h_{1 b_{1}}^{1}(t) \sum_{b_{2}=0}^{1} h_{2 b_{2}}^{1}(t) \cdots \sum_{b_{J}=0}^{1} h_{J^{1} b_{J}}^{1}(t) \\
\sum_{n=0}^{1} h_{\varsigma n}^{2}(t) \equiv \sum_{n_{1}=0}^{1} h_{1 n_{1}}^{2}(t) \sum_{n_{2}=0}^{1} h_{2 n_{2}}^{2}(t) \cdots \sum_{n_{J}=0}^{1} h_{J^{2} n_{J}}^{2}(t), \\
\vdots \\
\sum_{p=0}^{1} h_{\varsigma p}^{S}(t) \equiv \sum_{p_{1}=0}^{1} h_{1 p_{1}}^{S}(t) \sum_{p_{2}=0}^{1} h_{2 p_{2}}^{S}(t) \cdots \sum_{p_{J}=0}^{1} h_{J^{S} p_{J}}^{S}(t) \\
\sum_{\Omega} h_{\varsigma \Omega}^{\sigma}(t) \equiv \sum_{p=0}^{1} \ldots \sum_{n=0}^{1} \sum_{b=0}^{1} h_{\varsigma p}^{S}(t) \cdots h_{\varsigma n}^{2}(t) h_{\varsigma b}^{1}(t) \\
\varsigma=1,2, \ldots, J^{\sigma} ; \quad C_{\Omega}^{\sigma} \equiv G^{S} W^{S} \cdots G^{2} W^{2} G^{1} W^{1}
\end{gathered}
$$

and $b_{\varsigma}, n_{\varsigma}, p_{\varsigma}\left(\varsigma=1,2, \ldots, J^{\sigma}\right)$ represent the variables $\varphi$ of the $\varsigma$ th neuron of the first, second, and the Sth layer, respectively. Finally, according to (7), the dynamics of the NN model (13) can be rewritten as the following LDI state-space representation:

$$
\dot{X}(t)=\sum_{i=1}^{\phi} h_{i}(t) C_{i} \Lambda(t),
$$

where $h_{i}(t) \geq 0, \sum_{i=1}^{\phi} h_{i}(t)=1, \phi$ is a positive integer, and $C_{i}$ is a constant matrix with appropriate dimension associated with $C_{\Omega}^{\sigma}$. Moreover, the LDI state-space representation (15) can be rearranged as follows:

$$
\dot{X}(t)=\sum_{i=1}^{\phi} h_{i}(t)\left\{A_{i} X(t)+\sum_{k=1}^{m} \bar{A}_{i k} X\left(t-\tau_{k}\right)\right\},
$$

where $A_{i}$ and $\bar{A}_{i k}$ are the partitions of $C_{i}$ corresponding to the partitions of $\Lambda^{T}(t)$.

From the above, the NN models of the master and slave chaotic systems are described by the following LDI statespace representations (17) and (18), respectively:

$$
\begin{aligned}
& \text { Master: } \dot{X}(t)=\sum_{i=1}^{\phi} h_{i}(t)\left\{A_{i} X(t)+\sum_{k=1}^{m} \bar{A}_{i k} X\left(t-\tau_{k}\right)\right\} \\
& \text { Slave: } \dot{\hat{X}}(t)=\sum_{j=1}^{\phi} \widehat{h}_{j}(t)\left[\widehat{A}_{j} \widehat{X}(t)+\sum_{k=1}^{m} \widehat{\bar{A}}_{j k} \widehat{X}\left(t-\tau_{k}\right)\right] \\
& +B U(t) .
\end{aligned}
$$

2.3. Fuzzy Controller. According to the control scheme, a fuzzy controller is utilized to make the slave system synchronize with the master system.
The fuzzy controller takes the following form: Control Rule $l$ :

$$
\begin{aligned}
& \text { IF } e_{1}(t) \text { is } M_{l 1} \text { and } \cdots \text { and } e_{\delta}(t) \text { is } M_{l \delta} \\
& \text { THEN } U(t)=-K_{l} E(t),
\end{aligned}
$$

where $l=1,2, \ldots, \rho$, and $\rho$ is the number of IF-THEN rules of the fuzzy controller and $M_{l \eta}(\eta=1,2, \ldots, \delta)$ are the fuzzy sets. Hence, the final output of this fuzzy controller can be inferred as follows:

$$
\begin{aligned}
U(t) & =\frac{-\sum_{l=1}^{\rho} w_{l}(t) K_{l} E(t)}{\sum_{l=1}^{\rho} w_{l}(t)} \\
& =-\sum_{l=1}^{\rho} \bar{h}_{l}(t) K_{l} E(t)
\end{aligned}
$$

with $w_{l}(t) \equiv \prod_{\eta=1}^{\delta} M_{l \eta}\left(e_{\eta}(t)\right), \bar{h}_{l}(t) \equiv w_{l}(t) /\left(\sum_{l=1}^{\rho} w_{l}(t)\right)$, in which $M_{l \eta}\left(e_{\eta}(t)\right)$ is the grade of membership of $e_{\eta}(t)$ in $M_{l \eta}$. In this study, it is also assumed that $w_{l}(t) \geq 0(l=$ $1,2, \ldots, \rho)$ and $\sum_{l=1}^{\rho} w_{l}(t)>0$ for all $t$. Therefore, $\bar{h}_{l}(t) \geq 0$ and $\sum_{l=1}^{\rho} \bar{h}_{l}(t)=1$ for all $t$.

\section{Robustness Design of Chaotic Synchronization and Stability Analysis}

In this section, the synchronization of multiple time-delay chaotic (MTDC) systems is examined under the influence of modeling error.

3.1. Error Systems. From (1) and (2), the synchronization error is defined as $E(t) \equiv \widehat{X}(t)-X(t)=$ $\left[e_{1}(t), e_{2}(t), \ldots, e_{\delta}(t)\right]^{T}$ and then the dynamics of the error system under the fuzzy control (20) can be described as follows:

$$
\begin{aligned}
\dot{E}(t)= & \widehat{\Gamma}+\partial(t)-\Gamma \\
& +\sum_{i=1}^{\phi} \sum_{j=1}^{\phi} \sum_{l=1}^{\rho} h_{i}(t) \widehat{h}_{j}(t) \bar{h}_{l}(t) \\
& \times\left\{D_{i l} E(t)+\left(\widehat{A}_{j}-A_{i}\right) \widehat{X}(t)\right. \\
& +\sum_{k=1}^{m}\left(\widehat{\bar{A}}_{j k}-\bar{A}_{i k}\right) \widehat{X}\left(t-\tau_{k}\right) \\
& \left.+\sum_{k=1}^{m} \bar{A}_{i k} E\left(t-\tau_{k}\right)\right\} \\
& -\sum_{i=1}^{\phi} \sum_{j=1}^{\phi} \sum_{l=1}^{\rho} h_{i}(t) \widehat{h}_{j}(t) \bar{h}_{l}(t)
\end{aligned}
$$




$$
\begin{array}{r}
\times\left\{D_{i l} E(t)+\left(\widehat{A}_{j}-A_{i}\right) \widehat{X}(t)\right. \\
+\sum_{k=1}^{m}\left(\widehat{\bar{A}}_{j k}-\bar{A}_{i k}\right) \widehat{X}\left(t-\tau_{k}\right) \\
\left.+\sum_{k=1}^{m} \bar{A}_{i k} E\left(t-\tau_{k}\right)\right\} \\
=\sum_{i=1}^{\phi} \sum_{l=1}^{\rho} h_{i}(t) \bar{h}_{l}(t) \\
\times\left\{D_{i l} E(t)+\sum_{k=1}^{m} \bar{A}_{i k} E\left(t-\tau_{k}\right)\right\} \\
+\partial(t)+\Phi(t),
\end{array}
$$

where $D_{i l} \equiv A_{i}-B K_{l}$,

$$
\begin{gathered}
\widehat{\Gamma} \equiv f(\widehat{X}(t))+\sum_{k=1}^{m} H_{k}\left(\widehat{X}\left(t-\tau_{k}\right)\right)+U(t), \\
\Gamma \equiv f(X(t))+\sum_{k=1}^{m} H_{k}\left(X\left(t-\tau_{k}\right)\right)
\end{gathered}
$$

with

$$
\begin{gathered}
U(t)=-\sum_{l=1}^{\rho} \bar{h}_{l}(t) K_{l} E(t), \\
\Phi(t) \equiv \widehat{\Gamma}-\Gamma-\left\{\sum _ { i = 1 } ^ { \phi } \sum _ { l = 1 } ^ { \rho } h _ { i } ( t ) \overline { h } _ { l } ( t ) \left[D_{i l} E(t)\right.\right. \\
\left.\left.+\sum_{k=1}^{m} \bar{A}_{i k} E\left(t-\tau_{k}\right)\right]\right\} .
\end{gathered}
$$

Suppose that there exists a bounding matrix $\Delta Y_{i l}$ such that

$$
\|\Phi(t)\| \leq\left\|\sum_{i=1}^{\phi} \sum_{l=1}^{\rho} h_{i}(t) \bar{h}_{l}(t) \Delta Y_{i l} E(t)\right\|
$$

for the trajectory $E(t)$, and the bounding matrix $\Delta Y_{i l}$ can be described as follows:

$$
\Delta Y_{i l}=\kappa_{i l} Y,
$$

where $Y$ is the specified structured bounding matrix and $\left\|\kappa_{i l}\right\| \leq 1$ for $i=1,2, \ldots, \phi ; l=1,2, \ldots, \rho$. Equations (24) and (25) show that

$$
\begin{aligned}
\Phi^{T}(t) \Phi(t) \leq & {\left[\sum_{i=1}^{\phi} \sum_{l=1}^{\rho} h_{i}(t) \bar{h}_{l}(t) \Delta Y_{i l} E(t)\right]^{T} } \\
& \times\left[\sum_{i=1}^{\phi} \sum_{l=1}^{\rho} h_{i}(t) \bar{h}_{l}(t) \Delta Y_{i l} E(t)\right] \\
\leq & \sum_{i=1}^{\phi} \sum_{l=1}^{\rho} h_{i}(t) \bar{h}_{l}(t)\|Y E(t)\|\left\|\kappa_{i l}\right\| \\
& \times \sum_{i=1}^{\phi} \sum_{l=1}^{\rho} h_{i}(t) \bar{h}_{l}(t)\left\|\kappa_{i l}\right\|\|Y E(t)\| \\
\leq & {[Y E(t)]^{T}[Y E(t)] \leq E^{T}(t) Y^{T} Y E(t) . }
\end{aligned}
$$

Namely, $\Phi(t)$ is bounded by the specified structured bounding matrix $Y$.

Remark 1 (see [43]). The following simple example describes the procedures for determining $\kappa_{i l}$ and $Y$. First, assume that the possible bounds for all elements in $\Delta Y_{i l}$ are

$$
\Delta Y_{i l}=\left[\begin{array}{ccc}
\Delta y_{i l}^{11} & \Delta y_{i l}^{12} & \Delta y_{i l}^{13} \\
\Delta y_{i l}^{21} & \Delta y_{i l}^{22} & \Delta y_{i l}^{23} \\
\Delta y_{i l}^{31} & \Delta y_{i l}^{32} & \Delta y_{i l}^{33}
\end{array}\right],
$$

where $-y^{q s} \leq \Delta y_{i l}^{q s} \leq y^{q s}$ for some $y_{i l}^{q s}$ with $q, s=1,2,3$; $i=1,2, \ldots, \phi$; and $l=1,2, \ldots, \rho$.

A possible description for the bounding matrix $\Delta Y_{i l}$ is

$$
\Delta Y_{i l}=\left[\begin{array}{ccc}
\kappa_{i l}^{11} & 0 & 0 \\
0 & \kappa_{i l}^{22} & 0 \\
0 & 0 & \kappa_{i l}^{33}
\end{array}\right]\left[\begin{array}{lll}
y^{11} & y^{12} & y^{13} \\
y^{21} & y^{22} & y^{23} \\
y^{31} & y^{32} & y^{33}
\end{array}\right]=\kappa_{i l} Y,
$$

where $-1 \leq \kappa_{i l}^{q q} \leq 1$ for $q=1,2,3$. Notice that $\kappa_{i l}$ can be chosen by other forms as long as $\left\|\kappa_{i l}\right\| \leq 1$. Then, we check the validity of (24) in the simulation. If it is not satisfied, we can expand the bounds for all elements in $\Delta Y_{i l}$ and repeat the design procedure until (24) holds.

3.2. Delay-Dependent Stability Criterion for Exponential $H^{\infty}$ Synchronization. In this subsection, a delay-dependent criterion is proposed to guarantee the exponential stability of the error system described in (21). Moreover, in real physical systems, some noises or disturbances always exist that may cause instability and thereby destroy the synchronization performance. To reduce the effect of the external disturbance, an optimal $H^{\infty}$ scheme is used to design the fuzzy control so that the effect of external disturbance on control performance can be attenuated to a minimum level. In other words, in this study, the fuzzy controller (20) not only realizes exponential synchronization but also achieves the optimal $H^{\infty}$ control performance. 
Prior to the examination of the stability of the error system, some definitions and a lemma are given next.

Definition 2 (see [49]). The slave system (2) can exponentially synchronize with the master system (1) (i.e., the error system (21) is exponentially stable) if there exist two positive numbers $\alpha$ and $\beta$ such that the synchronization error satisfies

$$
\|E(t)\| \leq \alpha \exp \left(-\beta\left(t-t_{0}\right)\right), \quad \forall t \geq 0 .
$$

The positive number $\beta$ is called the exponential convergence rate.

Definition 3 (see [24-28]). The master system (1) and slave system (2) are said to be exponential $H^{\infty}$ synchronization if the following conditions are satisfied:

(i) in the case of $\partial(t)=0$, the error system (21) is exponentially stable,

(ii) under the zero initial conditions (i.e., $E(t)=0$ for $t \epsilon$ $\left[-\tau_{\max }, 0\right]$, in which $\tau_{\max }$ is the maximal value of $\tau_{k}$ 's) and a given constant $\gamma>0$, the following condition holds:

$$
\begin{aligned}
\Theta(E(t), \partial(t)) & =\int_{0}^{\infty} E^{T}(t) E(t) d t-\gamma^{2} \int_{0}^{\infty} \partial^{T}(t) \partial(t) d t \\
& \leq 0,
\end{aligned}
$$

where the parameter $\gamma$ is called the $H^{\infty}$-norm bound or the disturbance attenuation level. If the minimum $\gamma$ is found (i.e., the error system can reject the external disturbance as strongly as possible) to satisfy the above conditions, the fuzzy controller (20) is an optimal $H^{\infty}$ synchronizer [25].

Lemma 4 (see [50]). For the real matrices $A$ and $B$ with appropriate dimension, one has:

$$
A^{T} B+B^{T} A \leq \lambda A^{T} A+\lambda^{-1} B^{T} B,
$$

where $\lambda$ is a positive constant.

Theorem 5. For given positive constants $a$ and $n$, if there exist symmetric positive definite matrices $P, \psi_{k}$ and positive constant $c, \gamma$ such that the following inequalities hold, then the exponential $H^{\infty}$ synchronization with the disturbance attenuation $\gamma$ is guaranteed via the fuzzy controller (20):

$$
\begin{gathered}
\gamma>\sqrt{c m}, \\
\Delta_{i l} \equiv \sum_{k=1}^{m} \tau_{k} D_{i l}^{T} P+\sum_{k=1}^{m} \tau_{k} P D_{i l} \\
+\sum_{k=1}^{m} \tau_{k}^{2} P^{2}\left(c^{-1}+n^{-1}+m a^{-1}\right) \\
+\sum_{k=1}^{m} \psi_{k}+n m Y^{T} Y+I \\
<0, \\
\nabla_{i k} \equiv m a \bar{A}_{i k}^{T} \bar{A}_{i k}-\psi_{k}<0,
\end{gathered}
$$

where $D_{i l} \equiv A_{i}-B K_{l}$ for $i=1,2, \ldots, \phi ; k=1,2, \ldots, m$ and $l=1,2, \ldots, \rho$.

Proof. See the appendix.

Remark 6. Based on (24), $\Phi(t)$ is assumed to be bounded by the specified structured bounding matrix $Y$ and then the larger $\Phi(t)$ results in larger $Y$. Since the matrices $\Delta_{i l}$ must be negative definite to meet the stability condition (32b), the larger $Y$ will make Theorem 5 more difficult to satisfy.

Corollary 7. Equations (32b) and (32c) can be reformulated into LMIs via the following procedure.

By introducing the new variables $Q=P^{-1}, F_{l}=K_{l} Q$, and $\bar{\psi}_{k}=Q \psi_{k} Q,(32 \mathrm{~b})$ and (32c) can be rewritten as follows:

$$
\begin{gathered}
\sum_{k=1}^{m} \tau_{k}\left\{Q A_{i}^{T}-F_{l}^{T} B^{T}+A_{i} Q-B F_{l}\right\} \\
+\sum_{k=1}^{m} \tau_{k}^{2}\left(c^{-1}+n^{-1}+m a^{-1}\right) I \\
+\sum_{k=1}^{m} \bar{\psi}_{k}+n m Q Y^{T} Y Q+Q^{T} Q \\
<0, \\
m a Q^{T} \bar{A}_{i k}^{T} \bar{A}_{i k} Q-\bar{\psi}_{k}<0
\end{gathered}
$$

for $i=1,2, \ldots, \phi ; k=1,2, \ldots, m$, and $l=1,2, \ldots, \rho$. Based on Schur's complement [47], it is easy to show that the linear matrix inequalities in (33a) and (33b) are equivalent to the following LMIs in (34a) and (34b):

$$
\begin{aligned}
& {\left[\begin{array}{cc}
\Xi & Q Y^{T} \\
Y Q^{T} & -(n m)^{-1} I
\end{array}\right]<0,} \\
& {\left[\begin{array}{cc}
-\bar{\psi}_{k} & Q \bar{A}_{i k}^{T} \\
\bar{A}_{i k} Q & -(a m)^{-1} I
\end{array}\right]<0,}
\end{aligned}
$$

where

$$
\begin{aligned}
\Xi \equiv & \sum_{k=1}^{m} \tau_{k} Q A_{i}^{T}-\sum_{k=1}^{m} \tau_{k} F_{l}^{T} B^{T} \\
& +\sum_{k=1}^{m} \tau_{k} A_{i} Q-\sum_{k=1}^{m} \tau_{k} B F_{l} \\
& +\sum_{k=1}^{m} \tau_{k}^{2}\left(c^{-1}+n^{-1}+m a^{-1}\right) I \\
& +\sum_{k=1}^{m} \bar{\psi}_{k}+Q^{T} Q .
\end{aligned}
$$


Therefore, Theorem 5 can be transformed into an LMI problem, and efficient interior-point algorithms are now available in MATLAB LMI Solver to solve this problem.

Corollary 8 (see [51]). To verify the feasibility of solving the inequalities in (34a), (34b) by LMI Solver (MATLAB), the interior-point optimization techniques are utilized to compute feasible solutions. Such techniques require that the system of LMI is constrained to be strictly feasible; that is, the feasible set has a nonempty interior. For feasibility problems, the LMI Solver by feasp (feasp is the syntax used to test feasibility of a system of LMIs in MATLAB) is shown as follows:

Find $x$ such that the LMI $L(x)<0$

(in this study, (35) can be represented as (34a), (34b)) as

$$
\text { Minimize } t \text { subject to } L(x)<t \times I \text {. }
$$

From the above, the LMI constraint is always strictly feasible in $x, t$ and the original LMI (35) is feasible if and only if the global minimum $t$ min of (36) satisfies $t$ min $<0$. In other words, if $t$ min $<0$ will make (34a) and (34b) satisfied, then the stability conditions (32b) and (32c) in Theorem 5 can be met. Then, the obtained fuzzy controller (20) can exponentially stabilize the error system, and the $H^{\infty}$ control performance is achieved at the same time.

Remark 9. To reduce the computational burden, this study sets the positive constants $a$ and $n$ as unity.

Remark 10. It is an important issue to reduce the effect of external disturbances in synchronization process. The $H^{\infty}$ norm bound $\gamma$ is generally chosen as a positive small value less than unity for attenuation of disturbance. A smaller $\gamma$ is desirable as this yields better performance. However, a smaller $\gamma$ will result in a smaller $c$, making the stability conditions (32b) more difficult to satisfy.

Corollary 11. To achieve optimal $H^{\infty}$ exponential synchronization, the fuzzy control design is formulated as the following constrained optimization problem:

$$
\begin{array}{ll}
\text { minimize } & \gamma>\sqrt{c m} \\
\text { subject to } & Q=Q^{T}>0, \\
& \bar{\psi}_{k}=\bar{\psi}_{k}^{T}>0,(34 a) \text { and }(34 b) .
\end{array}
$$

More details to search the minimum $\gamma$ are given as follows.

The positive constant $c$ is minimized by the mincx function of MATLAB LMI Toolbox. Therefore, the minimum disturbance attenuation level $\gamma_{\min }>\sqrt{c_{\min } m}$ can be obtained.
The complete design procedure can be summarized in the following section.

\section{Algorithm}

Problem 1. Given two multiple time-delay chaotic systems with different initial conditions, how can a fuzzy controller be synthesized to realize the optimal $H^{\infty}$ exponential synchronization?

This problem can be solved according to the following steps.

Step 1. Construct the neural network (NN) models of the master system (1) and the slave system (2), respectively. On the basis of the interpolation method, the NN models are then converted into LDI state-space representations.

Step 2. According to the state-feedback control scheme, a fuzzy controller (20) is synthesized to exponentially stabilize the error system.

Step 3. Define the synchronization error $E(t)=\widehat{X}(t)-$ $X(t)$, and then the dynamics of the error system (21) can be obtained.

Step 4. Based on Corollary 11, the positive constant $c$ is minimized by the mincx function of MATLAB LMI Toolbox and then we have the minimum disturbance attenuation level.

Step 5. The matrices $P, F_{l}$, and $\bar{\psi}_{k}$ can be obtained with the minimum disturbance attenuation $\gamma_{\min }$.

\section{Numerical Example}

The following example is given to illustrate the effectiveness of the proposed algorithm.

Problem 2. The purpose of this example is to synthesize a fuzzy controller to achieve optimal $H^{\infty}$ exponential synchronization. Consider a pair of modified multiple time-delay Chen's chaotic systems in master-slave configuration, described as follows:

$$
\begin{aligned}
& \dot{x}_{1}(t)=35\left(x_{2}(t)-x_{1}(t)\right) \\
& \dot{x}_{2}(t)=-7 x_{1}(t-0.15)-x_{1}(t) x_{3}(t)+28 x_{2}(t-0.055) \\
& \dot{x}_{3}(t)=x_{1}(t) x_{2}(t)-3 x_{3}(t-0.12),
\end{aligned}
$$

$$
\begin{aligned}
\dot{\hat{x}}_{1}(t)= & 35\left(\widehat{x}_{2}(t)-\widehat{x}_{1}(t)\right)+\partial(t)+u_{1}(t), \\
\dot{\hat{x}}_{2}(t) & =-7 \widehat{x}_{1}(t-0.15)-\widehat{x}_{1}(t) \widehat{x}_{3}(t)+28 \widehat{x}_{2}(t-0.055) \\
& +\partial(t)+u_{2}(t), \\
\dot{x}_{3}(t)= & \widehat{x}_{1}(t) \widehat{x}_{2}(t)-3 \widehat{x}_{3}(t-0.12)+\partial(t)+u_{3}(t),
\end{aligned}
$$




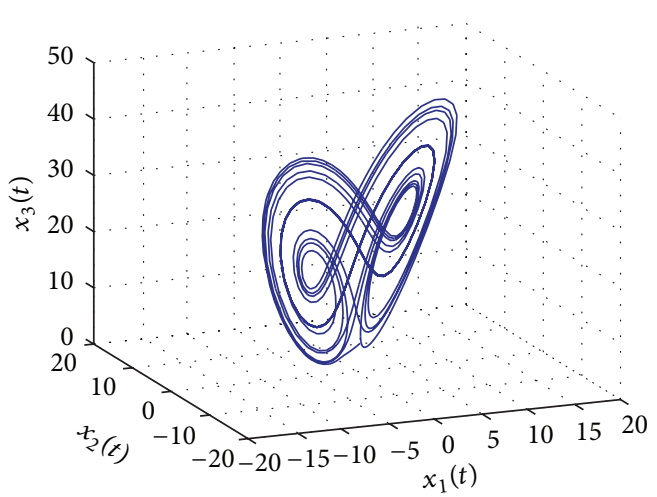

(a)

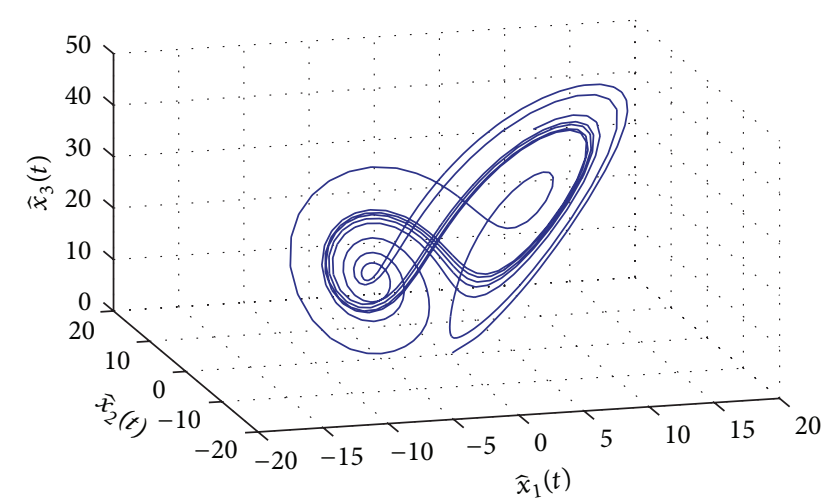

(b)

Figure 2: (a) Chaotic behavior of the master system (38). (b) Chaotic behavior of the slave system (39) without control.

where $\left[\begin{array}{lll}x_{1}(t) & x_{2}(t) & x_{3}(t)\end{array}\right]^{T}$ and $\left[\begin{array}{lll}\widehat{x}_{1}(t) & \widehat{x}_{2}(t) & \widehat{x}_{3}(t)\end{array}\right]^{T}$ are the state vectors of master and slave systems, respectively. Let the different initial conditions of master and slave systems be $\left[x_{1}(0)=-0.5 \quad x_{2}(0)=-2 \quad x_{3}(0)=6\right]$ and $\left[\widehat{x}_{1}(0)=1 \quad \hat{x}_{2}(0)=2 \quad \widehat{x}_{3}(0)=-1\right]$, and let the external disturbance $\partial(t)=0.5 \sin (2.3 t)$.

Figures 2(a) and 2(b) show the chaotic behaviors of the master (38) and slave (39) systems, respectively.

Solution. We can solve the above problem according to the following steps.

Step 1. Establish the NN models for master and slave systems via back propagation algorithm, respectively. First, the $\mathrm{NN}$ model to approximate the master chaotic system is constructed by $4-5-3$, and the transfer functions of all hidden neurons are chosen as follows:

$$
\begin{array}{r}
T\left(v_{\varsigma}^{\sigma}(t)\right)=\left\{\frac{2}{\left[1+\exp \left(-v_{\varsigma}^{\sigma}(t) / 0.5\right)\right]}-1\right\} \\
\text { for } \sigma=1,2 .
\end{array}
$$

On the other hand, the transfer functions of all output neurons are chosen as follows:

$$
T\left(v_{\varsigma}^{\sigma}(t)\right)=v_{\varsigma}^{\sigma}(t), \quad \text { for } \sigma=3
$$

After training, we can obtain the following the connection weights (the indices in $W_{\varsigma 9}^{\sigma}$ state that the weight of the $\sigma$ th layer in the NN model represents the connection to the $\varsigma$ th neuron from the 9 th source):

$$
\begin{aligned}
& W^{1} \\
& =\left[W_{\varsigma \vartheta}^{1}\right]=10^{-3} \\
& \times\left[\begin{array}{cccccccccccc}
-2.2596 & 1.9650 & -0.0363 & -0.0032 & -508.2983 & -548.8484 & 632.4534 & 0.0048 & 563.0571 & 790.8728 & -126.7520 & -0.0013 \\
-15.5783 & -27.7458 & -63.4663 & 1.0173 & 586.7615 & -331.7273 & 682.0762 & 0.4348 & 904.2274 & -284.3785 & 413.0475 & -4.4174 \\
70.0573 & -51.6754 & 2.1679 & 0.2317 & 807.5113 & -966.2829 & -831.8849 & -0.2983 & 217.1861 & -763.1395 & 788.9868 & 0.0089 \\
52.1919 & -18.8304 & -51.5188 & 1.8090 & 814.8914 & -164.8514 & 939.5783 & -1.5049 & -391.4165 & -277.6515 & -780.2455 & -3.8781
\end{array}\right], \\
& W^{2}=\left[W_{\varsigma 9}^{2}\right]=10^{-3} \times\left[\begin{array}{cccc}
90042.4610 & 265.5050 & 1364.4214 & -132.9508 \\
-99246.709 & 772.631 & -4313.1671 & 605.4461 \\
-221487.76 & -1715.2506 & -9271.4255 & -413.9966 \\
-6724.7569 & 0949.6135 & -624.7541 & -433.4421 \\
93499.239 & -735.4193 & 4088.0257 & -799.674
\end{array}\right] \text {, } \\
& W^{3}=\left[W_{\varsigma 9}^{3}\right]=10^{-3} \times\left[\begin{array}{ccccc}
349642.81 & 39165.768 & -13541.566 & -87345.175 & 56762.8100 \\
702.9312 & 844318.23 & 117376.51 & -667488.67 & 393028.9 \\
82032.708 & 5430424.3 & -402721.53 & -952938.89 & 5075605.6
\end{array}\right] .
\end{aligned}
$$

Then, the net inputs of the $\sigma$ th $(\sigma=1,2,3)$ layer are

$$
\begin{aligned}
v_{\varsigma}^{1}(t)= & W_{\varsigma 1}^{1} x_{1}(t)+W_{\varsigma 2}^{1} x_{2}(t)+W_{\varsigma 3}^{1} x_{3}(t) \\
& +W_{\varsigma 4}^{1} x_{1}(t-0.15)+W_{\varsigma 5}^{1} \cdot 0+W_{\varsigma 6}^{1} \cdot 0
\end{aligned}
$$

$$
\begin{array}{r}
+W_{\varsigma 7}^{1} \cdot 0+W_{\varsigma 8}^{1} x_{2}(t-0.055)+W_{\varsigma 9}^{1} \cdot 0 \\
+W_{\varsigma 10}^{1} \cdot 0+W_{\varsigma 11}^{1} \cdot 0+W_{\varsigma 12}^{1} x_{2}(t-0.12) \\
\varsigma=1,2,3,4
\end{array}
$$




$$
\begin{aligned}
& v_{\varsigma}^{2}(t)= W_{\varsigma 1}^{2} T\left(v_{1}^{1}(t)\right)+W_{\varsigma 2}^{2} T\left(v_{2}^{1}(t)\right) \\
&+W_{\varsigma 3}^{2} T\left(v_{3}^{1}(t)\right)+W_{\varsigma 4}^{2} T\left(v_{4}^{1}(t)\right), \\
& \varsigma=1,2,3,4,5, \\
& v_{\varsigma}^{3}(t)= W_{\varsigma 1}^{3} T\left(v_{1}^{2}(t)\right)+W_{\varsigma 2}^{3} T\left(v_{2}^{2}(t)\right) \\
&+W_{\varsigma 3}^{3} T\left(v_{3}^{2}(t)\right)+W_{\varsigma 4}^{3} T\left(v_{4}^{2}(t)\right) \\
&+W_{\varsigma 5}^{3} T\left(v_{5}^{2}(t)\right), \quad \varsigma=1,2,3, \\
& \dot{X}(t)= {\left[\begin{array}{l}
\dot{x}_{1}(t) \\
\dot{x}_{2}(t) \\
\dot{x}_{3}(t)
\end{array}\right]=\left[\begin{array}{l}
T\left(v_{1}^{3}(t)\right) \\
T\left(v_{2}^{3}(t)\right) \\
T\left(v_{3}^{3}(t)\right)
\end{array}\right] }
\end{aligned}
$$

(the symbol $v_{\varsigma}^{\sigma}$ denotes the net input of the $\varsigma$ th neuron of the $\sigma$ th layer in the NN model, and the indices $\sigma$ and $\varsigma$ shown in $h_{\varsigma \varphi}^{\sigma}(\varphi=1,2)$ indicate the same thing $)$.

According to (9), the minimum and the maximum of the derivative of each transfer function shown in (40) and (41) can be obtained as follows:

$$
\begin{gathered}
g_{\varsigma 0}^{1}=g_{\varsigma 0}^{2}=0, \quad g_{\varsigma 0}^{3}=1, \\
g_{\varsigma 1}^{1}=g_{\varsigma 1}^{2}=g_{\varsigma 1}^{3}=1, \quad \text { for } \varsigma=1,2, \ldots, J^{\sigma} .
\end{gathered}
$$

To simplify the notation, we let $g_{\varsigma 0}^{1}=g_{0}^{1}, g_{\varsigma 1}^{1}=g_{1}^{1}, g_{\varsigma 0}^{2}=$ $g_{0}^{2}, g_{\varsigma 1}^{2}=g_{1}^{2}, g_{\varsigma 0}^{3}=g_{0}^{3}$, and $g_{\varsigma 1}^{3}=g_{1}^{3}$. Then, based on the interpolation method, we have

$$
\begin{aligned}
\dot{x}_{1}(t)= & \sum_{d=0}^{1} h_{1 d}^{3}(t) g_{d}^{3} \sum_{\varsigma=1}^{5} W_{1 \varsigma}^{3} T\left(v_{\varsigma}^{2}(t)\right) \\
= & \sum_{d=0}^{1} h_{1 d}^{3}(t) g_{d}^{3} \sum_{\varsigma=1}^{5} W_{1 \varsigma}^{3}\left(h_{\varsigma 0}^{2}(t) g_{0}^{2}+h_{\varsigma 1}^{2}(t) g_{1}^{2}\right) \\
& \times \sum_{v=1}^{4} W_{\varsigma v}^{2} T\left(v_{v}^{1}(t)\right) \\
= & \sum_{d=0}^{1} h_{1 d}^{3}(t) g_{d}^{3} \sum_{\varsigma=1}^{5} W_{1 \varsigma}^{3}\left(h_{\varsigma 0}^{2}(t) g_{0}^{2}+h_{\varsigma 1}^{2}(t) g_{1}^{2}\right) \\
& \times \sum_{v=1}^{4} W_{\varsigma v}^{2}\left(h_{v 0}^{1}(t) g_{0}^{1}+h_{v 1}^{1}(t) g_{1}^{1}\right) v_{v}^{1}(t) \\
= & \sum_{d=0}^{1} h_{1 d}^{3}(t) g_{d}^{3} \sum_{\varsigma=1}^{5} W_{1 \varsigma}^{3}\left(h_{\varsigma 0}^{2}(t) g_{0}^{2}+h_{\varsigma 1}^{2}(t) g_{1}^{2}\right) \\
& \times \sum_{s=0}^{1} \sum_{p=0}^{1} \sum_{r=0}^{1} \sum_{o=0}^{1} h_{1 s}^{1}(t) h_{2 p}^{1}(t) h_{3 r}^{1}(t) h_{4 o}^{1}(t) \\
& \cdot\left(g_{s}^{1} W_{\varsigma 1}^{2} v_{1}^{1}(t)+g_{p}^{1} W_{\varsigma 2}^{2} v_{2}^{1}(t)\right.
\end{aligned}
$$

$$
\begin{aligned}
& \left.+g_{r}^{1} W_{\varsigma 3}^{2} v_{3}^{1}(t)+g_{o}^{1} W_{\varsigma 4}^{2} v_{4}^{1}(t)\right) \\
& =\sum_{d=0}^{1} h_{1 d}^{3}(t) g_{d}^{3} \\
& \times \sum_{c=0}^{1} \sum_{l=0}^{1} \sum_{k=0}^{1} \sum_{m=0}^{1} \sum_{n=0}^{1} h_{1 c}^{2}(t) h_{2 l}^{2}(t) \\
& \times h_{3 k}^{2}(t) h_{4 m}^{2}(t) h_{5 n}^{2}(t) \\
& \cdot \sum_{s=0}^{1} \sum_{p=0}^{1} \sum_{r=0}^{1} \sum_{o=0}^{1} h_{1 s}^{1}(t) h_{2 p}^{1}(t) h_{3 r}^{1}(t) h_{4 o}^{1}(t) \\
& \times\left(g_{c}^{2} W_{11}^{3} g_{s}^{1} W_{11}^{2} v_{1}^{1}(t)\right. \\
& +g_{c}^{2} W_{11}^{3} g_{p}^{1} W_{12}^{2} v_{2}^{1}(t) \\
& +g_{c}^{2} W_{11}^{3} g_{r}^{1} W_{13}^{2} v_{3}^{1}(t) \\
& +g_{c}^{2} W_{11}^{3} g_{o}^{1} W_{14}^{2} v_{4}^{1}(t) \\
& +g_{l}^{2} W_{12}^{3} g_{s}^{1} W_{21}^{2} v_{1}^{1}(t) \\
& +g_{l}^{2} W_{12}^{3} g_{p}^{1} W_{22}^{2} v_{2}^{1}(t) \\
& +g_{l}^{2} W_{12}^{3} g_{r}^{1} W_{23}^{2} v_{3}^{1}(t) \\
& +g_{l}^{2} W_{12}^{3} g_{o}^{1} W_{24}^{2} v_{4}^{1}(t) \\
& +g_{k}^{2} W_{13}^{3} g_{s}^{1} W_{31}^{2} v_{1}^{1}(t) \\
& +g_{k}^{2} W_{13}^{3} g_{p}^{1} W_{32}^{2} v_{2}^{1}(t) \\
& +g_{k}^{2} W_{13}^{3} g_{r}^{1} W_{33}^{2} v_{3}^{1}(t) \\
& +g_{k}^{2} W_{13}^{3} g_{o}^{1} W_{34}^{2} v_{4}^{1}(t) \\
& +g_{m}^{2} W_{14}^{3} g_{s}^{1} W_{41}^{2} v_{1}^{1}(t) \\
& +g_{m}^{2} W_{14}^{3} g_{p}^{1} W_{42}^{2} v_{2}^{1}(t) \\
& +g_{m}^{2} W_{14}^{3} g_{r}^{1} W_{43}^{2} v_{3}^{1}(t) \\
& +g_{m}^{2} W_{14}^{3} g_{o}^{1} W_{44}^{2} v_{4}^{1}(t) \\
& +g_{n}^{2} W_{15}^{3} g_{s}^{1} W_{51}^{2} v_{1}^{1}(t) \\
& +g_{n}^{2} W_{15}^{3} g_{p}^{1} W_{52}^{2} v_{2}^{1}(t) \\
& +g_{n}^{2} W_{15}^{3} g_{r}^{1} W_{53}^{2} v_{3}^{1}(t) \\
& \left.+g_{n}^{2} W_{15}^{3} g_{o}^{1} W_{54}^{2} v_{4}^{1}(t)\right) \\
& \dot{x}_{2}(t)=\sum_{e=0}^{1} h_{2 e}^{3}(t) g_{e}^{3} \sum_{\zeta=1}^{5} W_{2 \varsigma}^{3} T\left(v_{\zeta}^{2}(t)\right) \\
& =\sum_{e=0}^{1} h_{2 e}^{3}(t) g_{e}^{3} \sum_{\zeta=1}^{5} W_{2 \varsigma}^{3}\left(h_{\varsigma 0}^{2}(t) g_{0}^{2}+h_{\varsigma 1}^{2}(t) g_{1}^{2}\right)
\end{aligned}
$$




$$
\begin{aligned}
& \times \sum_{v=1}^{4} W_{\varsigma v}^{2} T\left(v_{v}^{1}(t)\right) \\
& =\sum_{e=0}^{1} h_{2 e}^{3}(t) g_{e}^{3} \sum_{\varsigma=1}^{5} W_{2 \varsigma}^{3}\left(h_{\varsigma 0}^{2}(t) g_{0}^{2}+h_{\varsigma 1}^{2}(t) g_{1}^{2}\right) \\
& \times \sum_{v=1}^{4} W_{\varsigma v}^{2}\left(h_{v 0}^{1}(t) g_{0}^{1}+h_{v 1}^{1}(t) g_{1}^{1}\right) v_{v}^{1}(t) \\
& =\sum_{e=0}^{1} h_{2 e}^{3}(t) g_{e}^{3} \sum_{\varsigma=1}^{5} W_{2 \varsigma}^{3}\left(h_{\varsigma 0}^{2}(t) g_{0}^{2}+h_{\varsigma 1}^{2}(t) g_{1}^{2}\right) \\
& \times \sum_{s=0}^{1} \sum_{p=0}^{1} \sum_{r=0}^{1} \sum_{o=0}^{1} h_{1 s}^{1}(t) h_{2 p}^{1}(t) h_{3 r}^{1}(t) h_{4 o}^{1}(t) \\
& \cdot\left(g_{s}^{1} W_{\varsigma 1}^{2} v_{1}^{1}(t)+g_{p}^{1} W_{\varsigma 2}^{2} v_{2}^{1}(t)\right. \\
& \left.+g_{r}^{1} W_{\varsigma 3}^{2} v_{3}^{1}(t)+g_{o}^{1} W_{\varsigma 4}^{2} v_{4}^{1}(t)\right) \\
& =\sum_{e=0}^{1} h_{2 e}^{3}(t) g_{e}^{3} \sum_{c=0}^{1} \sum_{l=0}^{1} \sum_{k=0}^{1} \sum_{m=0}^{1} \sum_{n=0}^{1} h_{1 c}^{2}(t) h_{2 l}^{2}(t) h_{3 k}^{2}(t) \\
& \times h_{4 m}^{2}(t) h_{5 n}^{2}(t) \\
& \cdot \sum_{s=0}^{1} \sum_{p=0}^{1} \sum_{r=0}^{1} \sum_{o=0}^{1} h_{1 s}^{1}(t) h_{2 p}^{1}(t) h_{3 r}^{1}(t) h_{4 o}^{1}(t) \\
& \times\left(g_{c}^{2} W_{21}^{3} g_{s}^{1} W_{11}^{2} v_{1}^{1}(t)\right. \\
& +g_{c}^{2} W_{21}^{3} g_{p}^{1} W_{12}^{2} v_{2}^{1}(t) \\
& +g_{c}^{2} W_{21}^{3} g_{r}^{1} W_{13}^{2} v_{3}^{1}(t) \\
& +g_{c}^{2} W_{21}^{3} g_{o}^{1} W_{14}^{2} v_{4}^{1}(t) \\
& +g_{l}^{2} W_{22}^{3} g_{s}^{1} W_{21}^{2} v_{1}^{1}(t) \\
& +g_{l}^{2} W_{22}^{3} g_{p}^{1} W_{22}^{2} v_{2}^{1}(t) \\
& +g_{l}^{2} W_{22}^{3} g_{r}^{1} W_{23}^{2} v_{3}^{1}(t) \\
& +g_{l}^{2} W_{22}^{3} g_{o}^{1} W_{24}^{2} v_{4}^{1}(t) \\
& +g_{k}^{2} W_{23}^{3} g_{s}^{1} W_{31}^{2} v_{1}^{1}(t) \\
& +g_{k}^{2} W_{23}^{3} g_{p}^{1} W_{32}^{2} v_{2}^{1}(t) \\
& +g_{k}^{2} W_{23}^{3} g_{r}^{1} W_{33}^{2} v_{3}^{1}(t) \\
& +g_{k}^{2} W_{23}^{3} g_{o}^{1} W_{34}^{2} v_{4}^{1}(t) \\
& +g_{m}^{2} W_{24}^{3} g_{s}^{1} W_{41}^{2} v_{1}^{1}(t) \\
& +g_{m}^{2} W_{24}^{3} g_{p}^{1} W_{42}^{2} v_{2}^{1}(t) \\
& +g_{m}^{2} W_{24}^{3} g_{r}^{1} W_{43}^{2} v_{3}^{1}(t)
\end{aligned}
$$

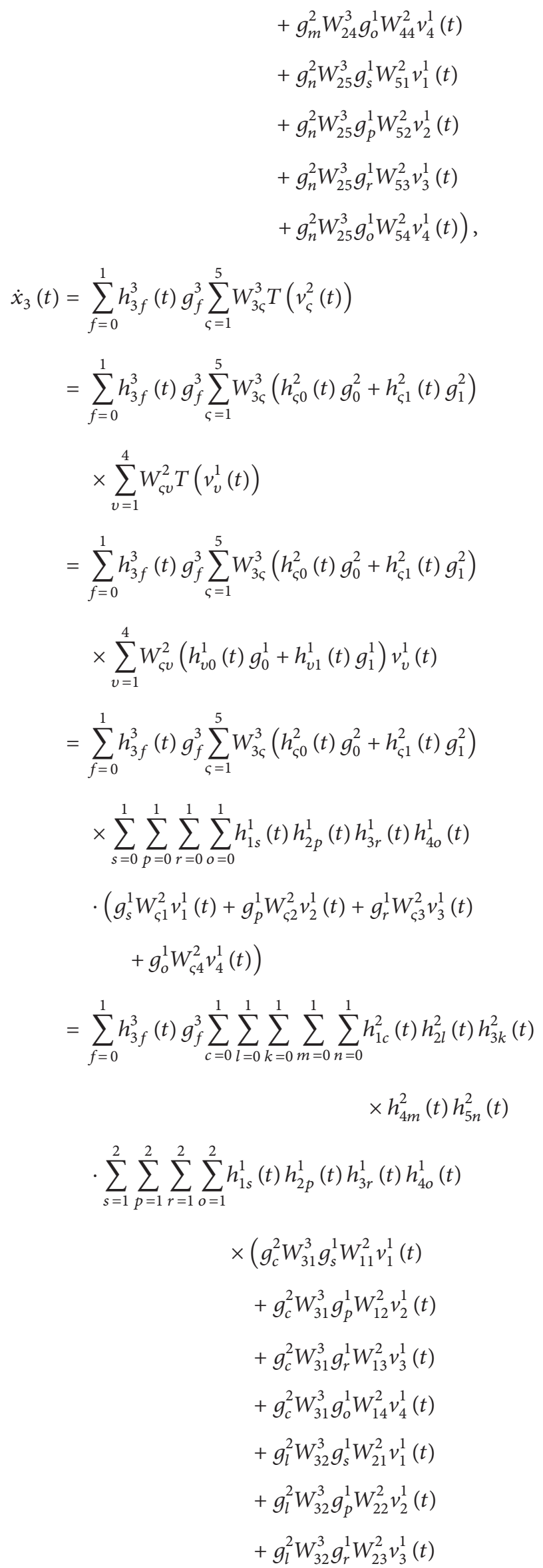




$$
\begin{aligned}
& +g_{l}^{2} W_{32}^{3} g_{o}^{1} W_{24}^{2} v_{4}^{1}(t) \\
& +g_{k}^{2} W_{33}^{3} g_{s}^{1} W_{31}^{2} v_{1}^{1}(t) \\
& +g_{k}^{2} W_{33}^{3} g_{p}^{1} W_{32}^{2} v_{2}^{1}(t) \\
& +g_{k}^{2} W_{33}^{3} g_{r}^{1} W_{33}^{2} v_{3}^{1}(t) \\
& +g_{k}^{2} W_{33}^{3} g_{o}^{1} W_{34}^{2} v_{4}^{1}(t) \\
& +g_{m}^{2} W_{34}^{3} g_{s}^{1} W_{41}^{2} v_{1}^{1}(t) \\
& +g_{m}^{2} W_{34}^{3} g_{p}^{1} W_{42}^{2} v_{2}^{1}(t) \\
& +g_{m}^{2} W_{34}^{3} g_{r}^{1} W_{43}^{2} v_{3}^{1}(t) \\
& +g_{m}^{2} W_{34}^{3} g_{o}^{1} W_{44}^{2} v_{4}^{1}(t) \\
& +g_{n}^{2} W_{35}^{3} g_{s}^{1} W_{51}^{2} v_{1}^{1}(t)
\end{aligned} \quad G^{1}=\left[\begin{array}{cccc}
g_{s}^{1} & 0 & 0 & 0 \\
0 & g_{p}^{1} & 0 & 0 \\
0 & 0 & g_{r}^{1} & 0 \\
0 & 0 & 0 & g_{o}^{1}
\end{array}\right],
$$$$
+g_{n}^{2} W_{35}^{3} g_{p}^{1} W_{52}^{2} v_{2}^{1}(t)
$$$$
+g_{n}^{2} W_{35}^{3} g_{r}^{1} W_{53}^{2} v_{3}^{1}(t)
$$$$
\left.+g_{n}^{2} W_{35}^{3} g_{o}^{1} W_{54}^{2} v_{4}^{1}(t)\right) \text {. }
$$

Then,

$$
\begin{array}{r}
E_{\text {defclkmnsproq }} \equiv G^{3} W^{3} G^{2} W^{2} G^{1} W^{1}=\left[\Upsilon_{\Re \aleph}\right]_{3 \times 12}, \\
\Re=1,2,3 ; \aleph=1,2 \ldots, 12 .
\end{array}
$$

Plugging (43a)-(43c) into (45) leads to

$$
\begin{aligned}
& \dot{X}(t)= \sum_{d=0}^{1} \sum_{e=0}^{1} \sum_{f=0}^{1} \sum_{c=0}^{1} \sum_{l=0}^{1} \sum_{k=0}^{1} \sum_{m=0}^{1} \sum_{n=0}^{1} \sum_{s=0}^{1} \sum_{p=0}^{1} \sum_{r=0}^{1} \sum_{o=0}^{1} h_{1 d}^{2}(t) h_{2 e}^{2}(t) h_{3 f}^{2}(t) h_{1 c}^{2}(t) h_{2 l}^{2}(t) h_{3 k}^{2}(t) h_{4 m}^{2}(t) h_{5 n}^{2}(t) \\
& \cdot h_{1 s}^{1}(t) h_{2 p}^{1}(t) h_{3 r}^{1}(t) h_{4 o}^{1}(t)\left\{A_{\text {defclkmnspro }} X(t)+\bar{A}_{\text {defclkmnspro1 }} X(t-0.15)\right. \\
&\left.+\bar{A}_{\text {defclkmnspro } 2} X(t-0.055)+\bar{A}_{\text {defclkmnspro3 }} X(t-0.12)\right\}
\end{aligned}
$$

where

$$
\begin{aligned}
& A_{\text {defclkmnspro }}=\left[\begin{array}{lll}
\Upsilon_{11} & \Upsilon_{12} & \Upsilon_{13} \\
\Upsilon_{21} & \Upsilon_{22} & \Upsilon_{23} \\
\Upsilon_{31} & \Upsilon_{32} & \Upsilon_{33}
\end{array}\right] \text {, } \\
& \bar{A}_{\text {defclkmnspro1 }}=\left[\begin{array}{lll}
\Upsilon_{14} & \Upsilon_{15} & \Upsilon_{16} \\
\Upsilon_{24} & \Upsilon_{25} & \Upsilon_{26} \\
\Upsilon_{34} & \Upsilon_{35} & \Upsilon_{36}
\end{array}\right] \text {, } \\
& \bar{A}_{\text {defclkmnspro2 }}=\left[\begin{array}{lll}
\Upsilon_{17} & \Upsilon_{18} & \Upsilon_{19} \\
\Upsilon_{27} & \Upsilon_{28} & \Upsilon_{29} \\
\Upsilon_{37} & \Upsilon_{38} & \Upsilon_{39}
\end{array}\right] \text {, } \\
& \bar{A}_{\text {deflkmnspro3 }}=\left[\begin{array}{lll}
\Upsilon_{110} & \Upsilon_{111} & \Upsilon_{112} \\
\Upsilon_{210} & \Upsilon_{211} & \Upsilon_{212} \\
\Upsilon_{310} & \Upsilon_{311} & \Upsilon_{312}
\end{array}\right] \text {, } \\
& X(t)=\left[\begin{array}{lll}
x_{1}(t) & x_{2}(t) & x_{3}(t)
\end{array}\right]^{T}, \\
& X(t-0.15)=\left[\begin{array}{lll}
x_{1}(t-0.15) & 0 & 0
\end{array}\right]^{T} \text {, } \\
& X(t-0.055)=\left[\begin{array}{lll}
0 & x_{2}(t-0.055) & 0
\end{array}\right]^{T}, \\
& X(t-0.12)=\left[\begin{array}{lll}
0 & 0 & x_{3}(t-0.12)
\end{array}\right]^{T} .
\end{aligned}
$$

Next, by renumbering the matrices shown in (48), the NN model of master system can be rewritten as the following LDI state-space representation:

$$
\dot{X}(t)=\sum_{i=1}^{4096} h_{i}(t)\left\{A_{i} X(t)+\sum_{k=1}^{3} \bar{A}_{i k} X\left(t-\tau_{k}\right)\right\},
$$

where $\tau_{1}=0.15, \tau_{2}=0.055, \tau_{3}=0.12$,

$$
\begin{aligned}
A_{1}= & A_{000000000000}, \ldots, A_{4065}=A_{111111111110}, \\
& A_{4096}=A_{111111111111}, \\
\bar{A}_{11}= & A_{0000000000001}, \ldots, \bar{A}_{40951}=A_{1111111111101}, \\
& \bar{A}_{40961}=A_{1111111111111}, \\
\bar{A}_{12}= & A_{0000000000002}, \ldots, \bar{A}_{40952}=A_{1111111111102}, \\
& \bar{A}_{40962}=A_{1111111111112}, \\
\bar{A}_{13}= & A_{0000000000003}, \ldots, \bar{A}_{40953}=A_{1111111111103}, \\
& \bar{A}_{40963}=A_{1111111111113} .
\end{aligned}
$$


Similarly, the connection weights of the NN model for the slave system are obtained as follows:

$$
\begin{aligned}
& \widehat{W}^{1}=\left[\widehat{W}_{\varsigma \vartheta}^{1}\right]=10^{-3} \\
& \times\left[\begin{array}{cccccccccccc}
133.4435 & -0079.5049 & -17.6211 & 3.3882 & -225.1168 & -64.0209 & -954.1527 & -3.4193 & 480.1857 & 754.7737 & 707.4367 & -2.159 \\
-62.4012 & 53.2513 & -4.9754 & 0.4651 & -383.4523 & -999.858 & 275.4848 & -0.25 & -190.4024 & 655.3 & 295.4735 & -0.2102 \\
-136.5871 & 80.423 & 17.6424 & -3.432 & -335.9 & 451.561 & -121.296 & 3.4809 & -897.175 & -102.7268 & 13.5665 & 2.1915 \\
46.5562 & 10.9547 & 24.1296 & -1.6127 & 863.1865 & -201.0341 & 183.5383 & 0.7656 & 896.0093 & 768.9494 & -847.9542 & 0.5831
\end{array}\right], \\
& \widehat{W}^{2}=\left[\widehat{\mathrm{W}}_{\varsigma \vartheta}^{2}\right]=10^{-3} \times\left[\begin{array}{cccc}
21614.815 & 1817.1715 & 17964.295 & -1655.6057 \\
-2215.2823 & 0.641 .7697 & -2.146 .7808 & 121.167 \\
-989.3919 & -1389.4418 & -1046.1459 & -410.0032 \\
128543.46 & -5108.3101 & 127475.58 & 2979.3142 \\
-24482.498 & 1165.7441 & -27137.819 & -2515.8115
\end{array}\right] \text {, } \\
& \widehat{W}^{3}=\left[\widehat{W}_{\varsigma 9}^{3}\right]=10^{-3} \times\left[\begin{array}{ccccc}
-215587.43 & 1177534.5 & 123780.4 & 18638.275 & 240168.12 \\
294714.01 & 312655.73 & -198923.25 & -55013.034 & -330253.83 \\
-1607287.8 & 4247988 & 2000786 & -48419.674 & 1579125.9
\end{array}\right] \text {. }
\end{aligned}
$$

Step 2. The procedures of constructing the NN model for the slave system are similar to those for the master system, and then we have the NN model of the slave system:

$$
\dot{\hat{X}}(t)=\sum_{j=1}^{4096} \widehat{h}_{j}(t)\left\{\widehat{A}_{j} \widehat{X}(t)+\sum_{k=1}^{3} \widehat{\bar{A}}_{j k} \widehat{X}\left(t-\tau_{k}\right)\right\}+B U(t)
$$

with $\tau_{1}=0.15, \tau_{2}=0.055, \tau_{3}=0.12$, and $B$ is a identity matrix. The responses of $\dot{X}(t)$ and $\dot{\hat{X}}(t)$ for original systems and NN models are shown in Figures 3(a) and 3(b).

Step 3. To synchronize the master and slave systems, a fuzzy controller is synthesized as follows:

Control Rule 1: IF $e_{1}(t)$ is $M_{1}$, THEN $U(t)=-K_{1} E(t)$,

Control Rule 2: IF $e_{1}(t)$ is $M_{2}, \quad$ THEN $U(t)=-K_{2} E(t)$,

where $M_{1}$ and $M_{2}$ are the membership functions for each $e_{1}$ (see Figure 4):

$$
\begin{gathered}
M_{1}\left(e_{1}\right)= \begin{cases}1, & e_{1} \geq 30 \\
\frac{e_{1}}{30}, & -30<e_{1}<30 \\
1, & e_{1} \leq-30,\end{cases} \\
M_{2}\left(e_{1}\right)=1-M_{1}\left(e_{1}\right) .
\end{gathered}
$$

According to (20), we have the overall fuzzy controller:

$$
U(t)=-\frac{\sum_{l=1}^{2} w_{l}(t) K_{l} E(t)}{\sum_{l=1}^{2} w_{l}(t)}=-\sum_{l=1}^{2} \bar{h}_{l}(t) K_{l l} E(t)
$$

with $w_{l}(t) \equiv M_{l}\left(e_{1}(t)\right), \bar{h}_{l}(t) \equiv w_{l}(t) /\left(\sum_{l=1}^{2} w_{l}(t)\right)$.
According to (21), the dynamics of the error system is obtained as follows:

$$
\begin{aligned}
\dot{E}(t)=\sum_{i=1}^{4096} \sum_{l=1}^{2} h_{i}(t) \bar{h}_{l}(t) & \\
& \times\left\{D_{i l} E(t)+\sum_{k=1}^{3} \bar{A}_{i k} E\left(t-\tau_{k}\right)\right\} \\
& +\partial(t)+\Phi(t),
\end{aligned}
$$

where $D_{i l} \equiv A_{i}-B K_{l}, \widehat{\Gamma} \equiv f(\widehat{X}(t))+\sum_{k=1}^{3} H_{k}\left(\widehat{X}\left(t-\tau_{k}\right)\right)+U(t)$ with $U(t)=-\sum_{l=1}^{2} h_{l}(t) K_{l} E(t)$,

$$
\begin{gathered}
\Gamma \equiv f(X(t))+\sum_{k=1}^{3} H_{k}\left(X\left(t-\tau_{k}\right)\right), \\
\Phi(t) \equiv \widehat{\Gamma}-\Gamma-\left\{\sum _ { i = 1 } ^ { 4 0 9 6 } \sum _ { l = 1 } ^ { 2 } h _ { i } ( t ) \overline { h } _ { l } ( t ) \left[D_{i l} E(t)\right.\right. \\
\left.\left.+\sum_{k=1}^{3} \bar{A}_{i k} E\left(t-\tau_{k}\right)\right]\right\} .
\end{gathered}
$$

Step 4. Based on (42), (48)-(57), the LMI in (34a) and (34b) can be solved via MATLAB LMI Toolbox. In accordance with Remark 1, the specified structured bounding matrices $Y$ and $\kappa_{i l}$ are set to be $Y=\left[\begin{array}{ccc}4600 & 0 & 0 \\ 0 & 4600 & 0 \\ 0 & 0 & 4600\end{array}\right], \kappa_{i l}=\left[\begin{array}{lll}1 & 0 & 0 \\ 0 & 1 & 0 \\ 0 & 0 & 1\end{array}\right]$. Based on Corollary 11, the positive constant $c$ is minimized by the mincx function of MATLAB LMI Toolbox: $c_{\min }=$ $5.3341 \times 10^{-15}$, and then we have the minimum disturbance attenuation level $\gamma_{\min }=1.0329 \times 10^{-7}$.

Step 5. The common solutions $P, F_{1}, F_{2}, \bar{\psi}_{1}, \bar{\psi}_{2}$, and $\bar{\psi}_{3}$ of the stability conditions (32b) and (32c) can be obtained with 

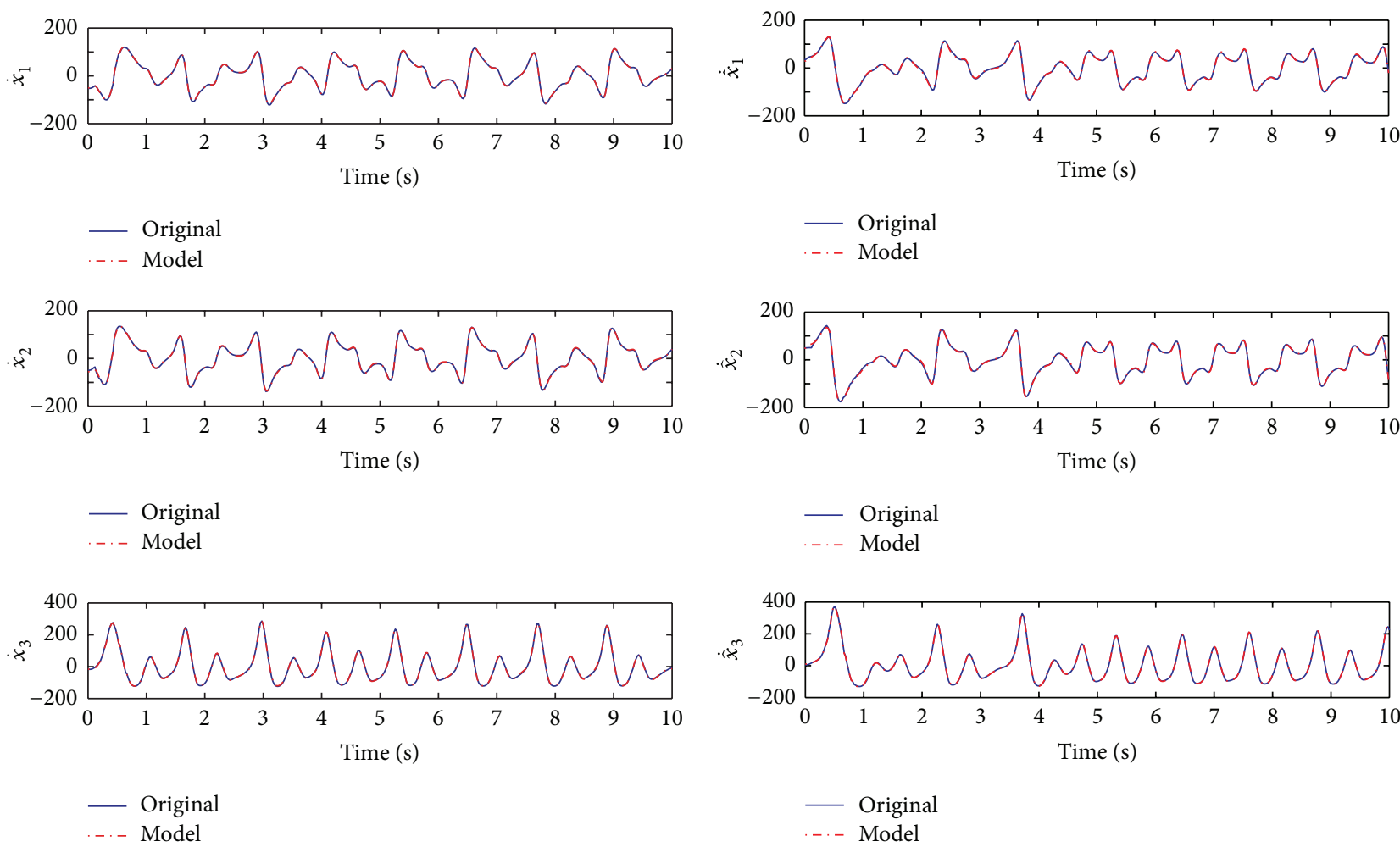

(a)

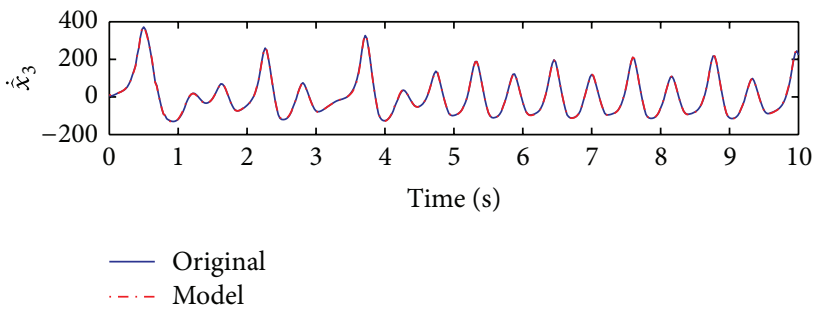

(b)

FIGURE 3: (a) The responses of $\dot{X}(t)$ for original system and NN model. (b) The responses of $\dot{X}(t)$ for original system and NN model.

the best value $t$ min of LMI Solver (MATLAB) of $-1.135048 \times$ $10^{-8}$ :

$$
\begin{aligned}
& P=10^{4} \times\left[\begin{array}{ccc}
4001.1183 & -268.3795 & 813.795 \\
-268.3795 & 2206.7761 & 778.8835 \\
813.795 & 778.8835 & 3191.7162
\end{array}\right], \\
& F_{1}=10^{-3} \times\left[\begin{array}{ccc}
0.1113 & 0.0257 & -0.0346 \\
0.0258 & 0.2094 & -0.0576 \\
-0.0347 & -0.0578 & 0.1515
\end{array}\right], \\
& F_{2}=10^{-3} \times\left[\begin{array}{ccc}
0.1113 & 0.0258 & -0.0347 \\
0.0258 & 0.2094 & -0.0576 \\
-0.0347 & -0.0576 & 0.1515
\end{array}\right]
\end{aligned}
$$

Furthermore, the resulting controller gains are

$$
\begin{aligned}
& K_{1}=\left[\begin{array}{ccc}
4103.0292 & -1.3653 & 0.8911 \\
1.3645 & 4103.0282 & 2.2966 \\
-0.8907 & -2.2952 & 4103.0288
\end{array}\right], \\
& K_{2}=\left[\begin{array}{ccc}
4103.0292 & -0.624 & -0.6759 \\
0.6233 & 4103.0282 & 1.7095 \\
0.6763 & -1.7081 & 4103.0288
\end{array}\right], \\
& \bar{\psi}_{1}=\bar{\psi}_{2}=\bar{\psi}_{3}=\left[\begin{array}{lll}
0.6228 & 0.0000 & 0.0000 \\
0.0000 & 0.6228 & 0.0001 \\
0.0000 & 0.0001 & 0.6228
\end{array}\right] .
\end{aligned}
$$

Figure 5 displays the state responses of both master and slave systems. The chaotic behaviors of the master and slave systems are shown in Figure 6. Moreover, Figure 7 illustrates the synchronization errors $\left(e_{1}, e_{2}\right.$, and $\left.e_{3}\right)$ which converge to zero. Furthermore, the assumption of $\|\Phi(t)\| \leq$ $\left\|\sum_{i=1}^{4096} \sum_{l=1}^{2} h_{i}(t) h_{l}(t) \Delta Y_{i l} E(t)\right\|$ is satisfied from the illustration shown in Figure 8.

\section{Conclusion}

In this study, an effective approach is proposed to realize the exponential synchronization of multiple time-delay chaotic (MTDC) systems, and the optimal $H^{\infty}$ performance is achieved at the same time. First, we employed a neural network (NN) model to approximate the MTDC system. A linear differential inclusion (LDI) state-space representation is then established for the dynamics of the NN model. Next, a delay-dependent stability criterion derived in terms of Lyapunov's direct method is presented to guarantee that the slave system can exponentially synchronize with the master system. Subsequently, the stability condition of this criterion is reformulated into a linear matrix inequality (LMI). Based on the Lyapunov stability theory and LMI approach, a fuzzy controller is synthesized to realize the exponential $H^{\infty}$ synchronization of the chaotic master-slave systems and reduce the $H^{\infty}$-norm from disturbance to synchronization error at the lowest level. Finally, simulation results demonstrate that 


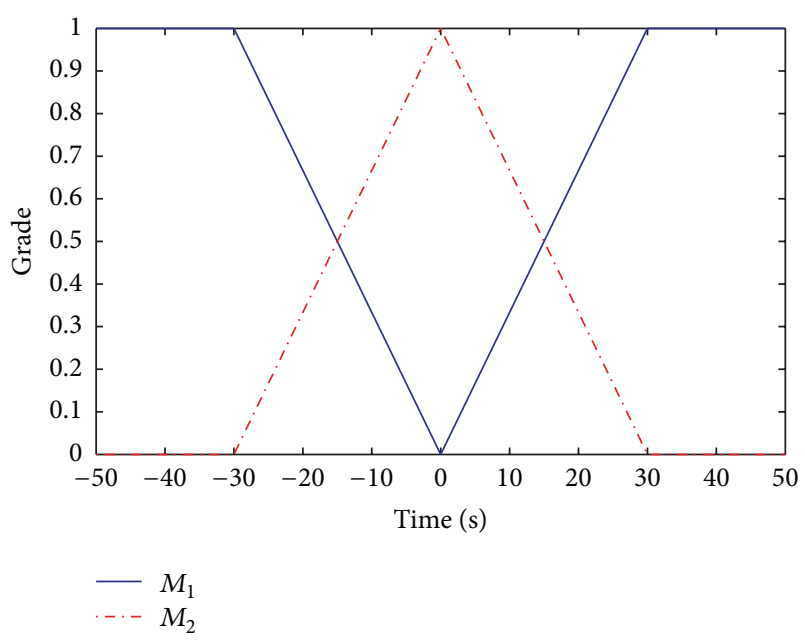

FIGURE 4: Membership functions of the fuzzy controller.
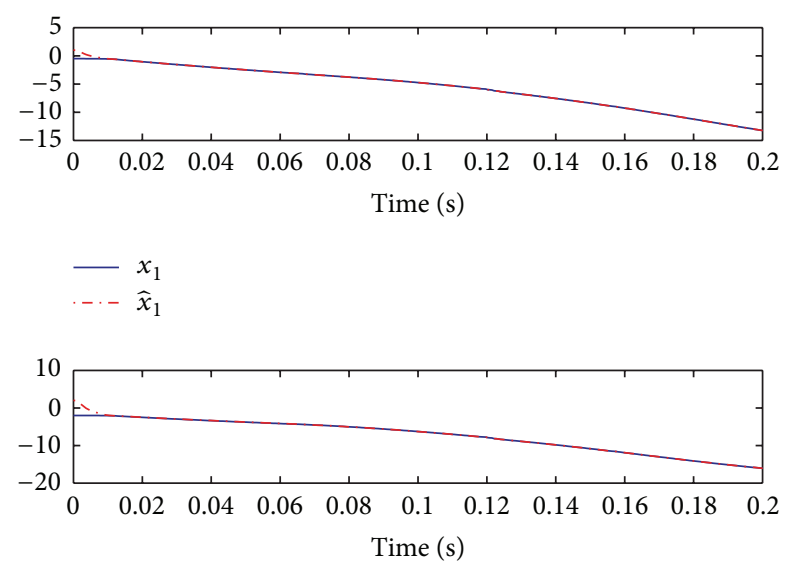

$$
\begin{array}{r}
x_{2} \\
-\cdots \\
\hat{x}_{2}
\end{array}
$$

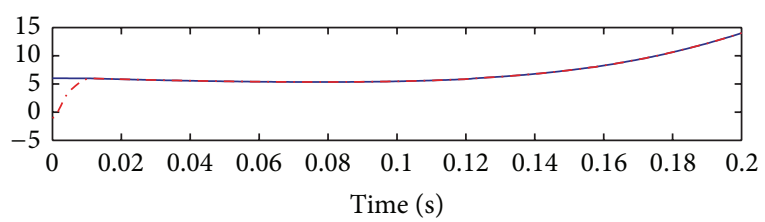

$$
\begin{array}{r}
x_{3} \\
--\hat{x}_{3}
\end{array}
$$

FIGURE 5: State responses of both master and slave systems.

the exponential $H^{\infty}$ synchronization of MTDC systems can be achieved by the designed fuzzy controller.

\section{Appendix}

\section{Proof of Theorem 5}

Let the Lyapunov function for the error system (21) be defined as

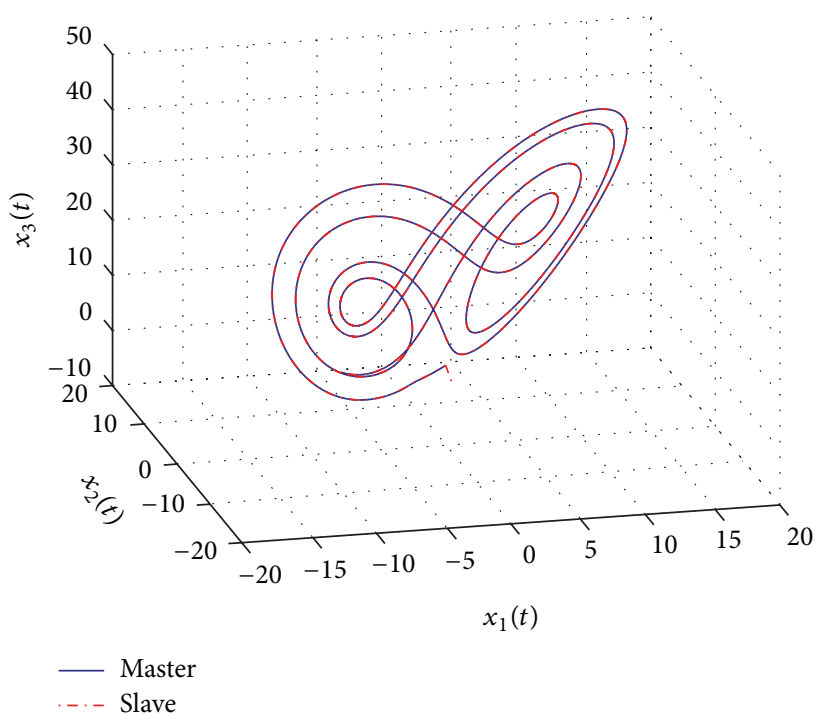

FIGURE 6: The chaotic behaviors of the master and the slave systems.
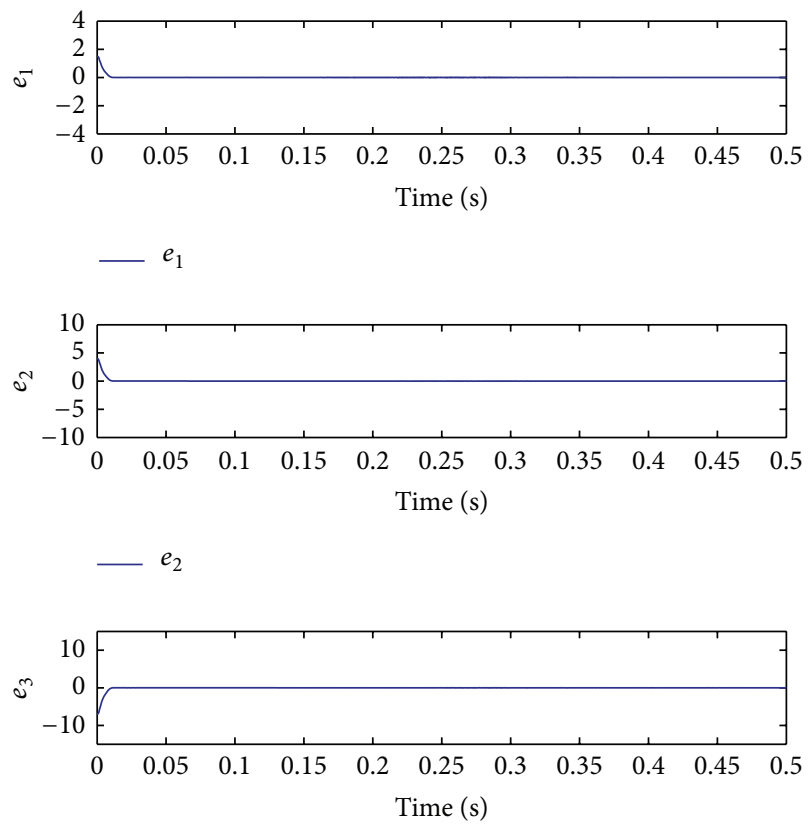

$-e_{3}$

Figure 7: State responses of the error system.

$$
\begin{aligned}
V(t)= & \sum_{k=1}^{m} E^{T}(t) \tau_{k} P E(t) \\
& +\sum_{k=1}^{m} \int_{0}^{\tau_{k}} E^{T}(t-\pi) \psi_{k} E(t-\pi) d \pi,
\end{aligned}
$$




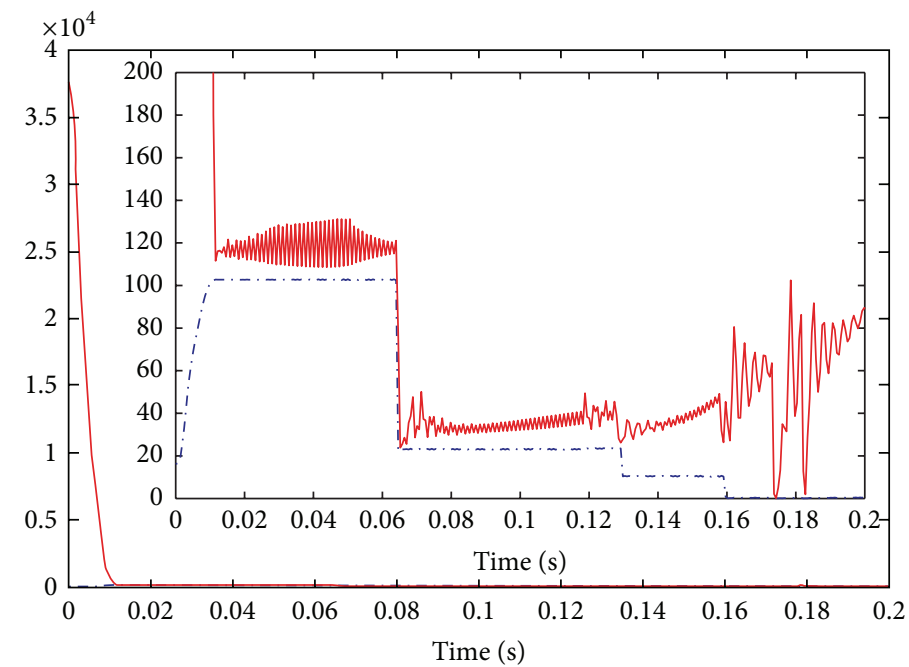

FIgure 8: Plots of $\|\Phi(t)\|$ (blue line) and $\left\|\sum_{i=1}^{4096} \sum_{l=1}^{2} h_{l}(t) h_{l}(t) \Delta Y_{i l} E(t)\right\|$ (red line).

where the weighting matrices $P=P^{T}>0$ and $\psi_{k}=\psi_{k}^{T}>0$. We then evaluate the time derivative of $V(t)$ on the trajectories of (21) to obtain

$$
\begin{aligned}
\dot{V}(t)= & \sum_{k=1}^{m} \tau_{k}\left[\dot{E}^{T}(t) P E(t)+E^{T}(t) P \dot{E}(t)\right] \\
& +\sum_{k=1}^{m}\left[E^{T}(t) \psi_{k} E(t)-E^{T}\left(t-\tau_{k}\right) \psi_{k} E\left(t-\tau_{k}\right)\right] \\
= & \sum_{k=1}^{m} \tau_{k}\left\{\sum_{i=1}^{\phi} \sum_{l=1}^{\rho} h_{i}(t) \bar{h}_{l}(t)\right. \\
& \times\left[D_{i l} E(t)+\sum_{d=1}^{m} \bar{A}_{i d} E\left(t-\tau_{d}\right)\right] \\
& +\partial(t)+\Phi(t)\}^{T} P E(t) \\
& +\sum_{k=1}^{m} \tau_{k} E^{T}(t) P \\
& \times\left\{\sum _ { i = 1 } ^ { \phi } \sum _ { l = 1 } ^ { \rho } h _ { i } ( t ) \overline { h } _ { l } ( t ) \left[D_{i l} E(t)\right.\right. \\
& +\sum_{k=1}^{m}\left[E^{T}(t) \psi_{k} E(t)-E_{i d}^{T} E\left(t-\tau_{d}\right)\right.
\end{aligned}
$$

$$
\begin{gathered}
=\sum_{k=1}^{m} \sum_{i=1}^{\phi} \sum_{l=1}^{\rho} h_{i}(t) \bar{h}_{l}(t) E^{T}(t) \\
\times\left[\tau_{k} D_{i l}^{T} P+\tau_{k} P D_{i l}+\psi_{k}\right] E(t) \\
+\sum_{k=1}^{m} \sum_{i=1}^{\phi} \sum_{d=1}^{m} h_{i}(t)\left[E^{T}\left(t-\tau_{d}\right) \tau_{k} \bar{A}_{i d}^{T} P E(t)\right. \\
\left.+E^{T}(t) \tau_{k} P \bar{A}_{i d} E\left(t-\tau_{d}\right)\right] \\
+\sum_{k=1}^{m}\left[\partial^{T}(t) \tau_{k} P E(t)+E^{T}(t) \tau_{k} P \partial(t)\right. \\
\left.+\Phi^{T}(t) \tau_{k} P E(t)+E^{T}(t) \tau_{k} P \Phi(t)\right] \\
-\sum_{k=1}^{m}\left[E^{T}\left(t-\tau_{k}\right) \psi_{k} E\left(t-\tau_{k}\right)\right] .
\end{gathered}
$$

Based on Lemma 4 and (A.2), we have

$$
\begin{gathered}
\dot{V}(t) \leq \sum_{k=1}^{m} \sum_{i=1}^{\phi} \sum_{l=1}^{\rho} h_{i}(t) \bar{h}_{l}(t) E^{T}(t) \\
\quad \times\left[\tau_{k} D_{i l}^{T} P+\tau_{k} P D_{i l}+\psi_{k}\right] E(t) \\
+\sum_{k=1}^{m} \sum_{i=1}^{\phi} \sum_{d=1}^{m} h_{i}(t)\left[a E^{T}\left(t-\tau_{d}\right) \bar{A}_{i d}^{T} \bar{A}_{i d} E\left(t-\tau_{d}\right)\right. \\
\left.+a^{-1} E^{T}(t) \tau_{k}^{2} P^{2} E(t)\right] \\
+\sum_{k=1}^{m}\left[c \partial^{T}(t) \partial(t)+c^{-1} E^{T}(t) \tau_{k}^{2} P^{2} E(t)\right. \\
\left.\quad+n \Phi^{T}(t) \Phi(t)+n^{-1} E^{T}(t) \tau_{k}^{2} P^{2} E(t)\right] \\
-\sum_{k=1}^{m}\left[E^{T}\left(t-\tau_{k}\right) \psi_{k} E\left(t-\tau_{k}\right)\right]
\end{gathered}
$$




$$
\begin{gathered}
\leq \sum_{k=1}^{m} \sum_{i=1}^{\phi} \sum_{l=1}^{\rho} h_{i}(t) \bar{h}_{l}(t) E^{T}(t) \\
\quad \times\left[\tau_{k} D_{i l}^{T} P+\tau_{k} P D_{i l}+\psi_{k}\right] E(t) \\
+\sum_{k=1}^{m} \sum_{i=1}^{\phi} \sum_{d=1}^{m} h_{i}(t)\left[a E^{T}\left(t-\tau_{d}\right) \bar{A}_{i d}^{T} \bar{A}_{i d} E\left(t-\tau_{d}\right)\right. \\
\left.+a^{-1} E^{T}(t) \tau_{k}^{2} P^{2} E(t)\right] \\
+\sum_{k=1}^{m}\left[c \partial^{T}(t) \partial(t)+c^{-1} E^{T}(t) \tau_{k}^{2} P^{2} E(t)\right. \\
+n E^{T}(t) Y^{T} Y E(t) \\
\left.+n^{-1} E^{T}(t) \tau_{k}^{2} P^{2} E(t)\right] \\
-\sum_{k=1}^{m}\left[E^{T}\left(t-\tau_{k}\right) \psi_{k} E\left(t-\tau_{k}\right)\right]
\end{gathered}
$$$$
=\sum_{i=1}^{\phi} \sum_{l=1}^{\rho} h_{i}(t) \bar{h}_{l}(t) E^{T}(t)
$$$$
\times\left[\sum_{k=1}^{m} \tau_{k} D_{i l}^{T} P+\sum_{k=1}^{m} \tau_{k} P D_{i l}\right.
$$$$
+\sum_{k=1}^{m} \tau_{k}^{2} P^{2}\left(c^{-1}+n^{-1}+m a^{-1}\right)
$$

$$
\left.+\sum_{k=1}^{m} \psi_{k}+n m Y^{T} Y\right] E(t)
$$$$
+\sum_{k=1}^{m} \sum_{i=1}^{\phi} h_{i}(t) E^{T}\left(t-\tau_{k}\right)\left[m a \bar{A}_{i k}^{T} \bar{A}_{i k}-\psi_{k}\right]
$$$$
\times E\left(t-\tau_{k}\right)+c m \partial^{T}(t) \partial(t) .
$$

From (A.5), we have

$$
\begin{gathered}
\dot{V}(t)+E^{T}(t) E(t)-\gamma^{2} \partial^{T}(t) \partial(t) \\
\leq \sum_{i=1}^{\phi} \sum_{l=1}^{\rho} h_{i}(t) \bar{h}_{l}(t) E^{T}(t) \\
\times\left[\sum_{k=1}^{m} \tau_{k} D_{i l}^{T} P+\sum_{k=1}^{m} \tau_{k} P D_{i l}\right. \\
+\sum_{k=1}^{m} \tau_{k}^{2} P^{2}\left(c^{-1}+n^{-1}+m a^{-1}\right) \\
\left.+\sum_{k=1}^{m} \psi_{k}+n m Y^{T} Y\right] \\
\times E(t)+E^{T}(t) E(t)
\end{gathered}
$$

$$
\begin{aligned}
+\sum_{k=1}^{m} \sum_{i=1}^{\phi} h_{i}(t) E^{T}\left(t-\tau_{k}\right)\left[m a \bar{A}_{i k}^{T} \bar{A}_{i k}-\psi_{k}\right] \\
\times E\left(t-\tau_{k}\right)+c m \partial^{T}(t) \partial(t)-\gamma^{2} \partial^{T}(t) \partial(t)
\end{aligned}
$$

$$
\begin{aligned}
&=\sum_{i=1}^{\phi} \sum_{l=1}^{\rho} h_{i}(t) \bar{h}_{l}(t) E^{T}(t) \\
& \times\left[\sum_{k=1}^{m} \tau_{k} D_{i l}^{T} P+\sum_{k=1}^{m} \tau_{k} P D_{i l}\right. \\
&+\sum_{k=1}^{m} \tau_{k}^{2} P^{2}\left(c^{-1}+n^{-1}+m a^{-1}\right) \\
&\left.+\sum_{k=1}^{m} \psi_{k}+n m Y^{T} Y+I\right] E(t)
\end{aligned}
$$

$+\sum_{k=1}^{m} \sum_{i=1}^{\phi} h_{i}(t) E^{T}\left(t-\tau_{k}\right)$

$$
\begin{aligned}
& \times\left[m a \bar{A}_{i k}^{T} \bar{A}_{i k}-\psi_{k}\right] E\left(t-\tau_{k}\right) \\
& +c m \partial^{T}(t) \partial(t)-\gamma^{2} \partial^{T}(t) \partial(t)
\end{aligned}
$$$$
=\sum_{i=1}^{\phi} \sum_{l=1}^{\rho} h_{i}(t) \bar{h}_{l}(t) E^{T}(t) \Delta_{i l} E(t)
$$$$
+\sum_{i=1}^{\phi} \sum_{k=1}^{m} h_{i}(t) E^{T}\left(t-\tau_{k}\right) \nabla_{i k} E\left(t-\tau_{k}\right)
$$$$
+\left(c m-\gamma^{2}\right) \partial^{T}(t) \partial(t)
$$$$
\leq \sum_{i=1}^{\phi} \sum_{l=1}^{\rho} h_{i}(t) \bar{h}_{l}(t) \lambda_{\max }\left(\Delta_{i l}\right) E^{T}(t) E(t)
$$$$
+\sum_{i=1}^{\phi} \sum_{k=1}^{m} h_{i}(t) \lambda_{\max }\left(\nabla_{i k}\right) E^{T}\left(t-\tau_{k}\right) E\left(t-\tau_{k}\right)
$$$$
+\left(c m-\gamma^{2}\right) \partial^{T}(t) \partial(t)
$$

$<0$,

where

$$
\begin{aligned}
\Delta_{i l} \equiv & \sum_{k=1}^{m} \tau_{k} D_{i l}^{T} P+\sum_{k=1}^{m} \tau_{k} P D_{i l} \\
& +\sum_{k=1}^{m} \tau_{k}^{2} P^{2}\left(c^{-1}+n^{-1}+m a^{-1}\right) \\
& +\sum_{k=1}^{m} \psi_{k}+n m Y^{T} Y+I \quad(\operatorname{see}(32 \mathrm{~b})) \\
\nabla_{i k} \equiv & m a \bar{A}_{i k}^{T} \bar{A}_{i k}-\psi_{k} \quad(\operatorname{see}(32 \mathrm{c})) .
\end{aligned}
$$


Integrating (A.6a), (A.6b), and (A.6c) from $t=0$ to $t=$ $\infty$, the following inequality is obtained as

$$
\begin{gathered}
V(\infty)-V(0)+\int_{0}^{\infty} E^{T}(t) E(t) d t \\
-\gamma^{2} \int_{0}^{\infty} \partial^{T}(t) \partial(t) d t
\end{gathered}
$$

$$
\leq 0 \text {. }
$$

With zero initial conditions (i.e., $E(t) \equiv 0$ for $t \in$ $\left.\left[-\tau_{\max }, 0\right]\right)$, we have

$$
\int_{0}^{\infty} E^{T}(t) E(t) d t \leq \gamma^{2} \int_{0}^{\infty} \partial^{T}(t) \partial(t) d t .
$$

That is, (30) and the $H^{\infty}$ control performance are achieved with a prescribed attenuation $\gamma$.

Since

$$
\begin{aligned}
& \sum_{k=1}^{m} \tau_{k} \lambda_{\min }(P) E^{T}(t) E(t) \leq \sum_{k=1}^{m} \tau_{k} E^{T}(t) P E(t) \\
&=V(t)-\sum_{k=1}^{m} \int_{0}^{\tau_{k}} E^{T}(t-\pi) \\
& \quad \times \psi_{k} E(t-\pi) d \pi \\
&<V(t) \quad(\text { from (A.1)) }
\end{aligned}
$$

we can get the following inequality from (A.6a), (A.6b), and (A.6c):

$$
\begin{aligned}
\dot{V}(t) & +E^{T}(t) E(t)-\gamma^{2} \partial^{T}(t) \partial(t) \\
& <\sum_{i=1}^{\phi} \sum_{l=1}^{\rho} h_{i}(t) \bar{h}_{l}(t) \frac{\lambda_{\max }\left(\Delta_{i l}\right)}{\sum_{k=1}^{m} \tau_{k} \lambda_{\min }(P)} V(t) \\
& <0 .
\end{aligned}
$$

Then, we can obtain

$$
\left.V(t)\right|_{\partial(t)=0}<V\left(t_{0}\right) \exp \bar{\beta}\left(t-t_{0}\right),
$$

where

$$
\bar{\beta}=\sum_{i=1}^{\phi} \sum_{l=1}^{\rho} h_{i}(t) \bar{h}_{l}(t)\left[\frac{\lambda_{\max }\left(\Delta_{i l}\right)}{\sum_{k=1}^{m} \tau_{k} \lambda_{\min }(P)}\right] .
$$

From (A.1) and (A.12), we have

$$
\begin{aligned}
\sum_{k=1}^{m} \tau_{k} \lambda_{\min }(P) E^{T}(t) E(t) \\
\leq \sum_{k=1}^{m} E^{T}(t) \tau_{k} P E(t) \\
<V\left(t_{0}\right) \exp \bar{\beta}\left(t-t_{0}\right) \\
\quad-\sum_{k=1}^{m} \int_{0}^{\tau_{k}} E^{T}(t-\pi) \psi_{k} E(t-\pi) d \pi \\
<V\left(t_{0}\right) \exp \bar{\beta}\left(t-t_{0}\right) .
\end{aligned}
$$

That is, $\|E(t)\|^{2} \leq\left(V\left(t_{0}\right) /\left(\sum_{k=1}^{m} \tau_{k} \lambda_{\min }(P)\right)\right) \exp \bar{\beta}\left(t-t_{0}\right)$. Consequently, we conclude that

$$
\begin{aligned}
& \|E(t)\| \leq \alpha \exp \left(-\beta\left(t-t_{0}\right)\right) \\
& \text { with } \alpha \equiv \sqrt{\frac{V\left(t_{0}\right)}{\sum_{k=1}^{m} \tau_{k} \lambda_{\text {min }}(P)}}>0, \beta \equiv-\frac{1}{2} \bar{\beta}>0 .
\end{aligned}
$$

Therefore, based on Definition 2, the error system (21) with the fuzzy controller (20) is exponentially stable for $\partial(t)=0$.

\section{References}

[1] L. Denis-Vidal, C. Jauberthie, and G. Joly-Blanchard, "Identifiability of a nonlinear delayed-differential aerospace model," IEEE Transactions on Automatic Control, vol. 51, no. 1, pp. 154-158, 2006.

[2] R. Loxton, K. L. Teo, and V. Rehbock, "An optimization approach to state-delay identification," IEEE Transactions on Automatic Control, vol. 55, no. 9, pp. 2113-2119, 2010.

[3] L. Y. Wang, W. H. Gui, K. L. Teo, R. Loxton, and C. H. Yang, "Optimal control problems arising in the zinc sulphate electrolyte purification process," Journal of Global Optimization, vol. 54, no. 2, pp. 307-323, 2012.

[4] Q. Chai, R. Loxton, K. L. Teo, and C. Yang, "A unified parameter identification method for nonlinear time-delay systems," Journal of Industrial and Management Optimization, vol. 9, pp. 471486, 2013.

[5] Q. Chai, R. Loxton, K. L. Teo, and C. Yang, "Time-delay estimation for nonlinear systems with piecewise-constant input," Applied Mathematics and Computation, vol. 219, no. 17, pp. 9543-9560, 2013.

[6] M. Mackey and L. Glass, "Oscillation and chaos in physiological control systems," Science, vol. 197, no. 4300, pp. 287-289, 1977.

[7] H. O. Wang and E. H. Abed, "Bifurcation control of a chaotic system," Automatica, vol. 31, no. 9, pp. 1213-1226, 1995.

[8] L. Kocarev and U. Parlitz, "General approach for chaotic synchronization with applications to communication," Physical Review Letters, vol. 74, no. 25, pp. 5028-5031, 1995.

[9] J.-Q. Zhu, H. Takakubo, and K. Shono, "Observation and analysis of chaos with digitalizing measure in a CMOS mapping system," IEEE Transactions on Circuits and Systems I, vol. 43, no. 6, pp. 444-452, 1996.

[10] G. Poddar, K. Chakrabarty, and S. Banerjee, "Control of chaos in DC-DC converters," IEEE Transactions on Circuits and Systems I, vol. 45, no. 6, pp. 672-676, 1998.

[11] S. L. Lin and P. C. Tung, "A new method for chaos control in communication systems," Chaos, Solitons and Fractals, vol. 42, no. 5, pp. 3234-3241, 2009.

[12] Q. Chai, R. Loxton, K. L. Teo, and C. Yang, "A class of optimal state-delay control problems," Nonlinear Analysis. Real World Applications, vol. 14, no. 3, pp. 1536-1550, 2013.

[13] L. M. Pecora and T. L. Carroll, "Synchronization in chaotic systems," Physical Review Letters, vol. 64, no. 8, pp. 821-824, 1990.

[14] S. Li, W. Xu, and R. Li, "Synchronization of two different chaotic systems with unknown parameters," Physics Letters A, vol. 361, no. 1-2, pp. 98-102, 2007.

[15] J. H. Kim, C. H. Hyun, E. Kim, and M. Park, "Adaptive synchronization of uncertain chaotic systems based on T-S 
fuzzy model," IEEE Transactions on Fuzzy Systems, vol. 15, no. 3, pp. 359-369, 2007.

[16] H.-T. Yau and J.-J. Yan, "Chaos synchronization of different chaotic systems subjected to input nonlinearity," Applied Mathematics and Computation, vol. 197, no. 2, pp. 775-788, 2008.

[17] S. Jankowski, A. Londei, A. Lozowski, and C. Mazur, "Synchronization and control in a cellular neural network of chaotic units by local pinnings," International Journal of Circuit Theory and Applications, vol. 24, no. 3, pp. 275-281, 1996.

[18] G. Rangarajan and M. Ding, "Stability of synchronized chaos in coupled dynamical systems," Physics Letters A, vol. 296, no. 4-5, pp. 204-209, 2002.

[19] H. J. Yu and Y. Liu, "Chaotic synchronization based on stability criterion of linear systems," Physics Letters A, vol. 314, no. 4, pp. 292-298, 2003.

[20] G. Chen, J. Zhou, and Z. Liu, "Global synchronization of coupled delayed neural networks and applications to chaotic CNN models," International Journal of Bifurcation and Chaos, vol. 14, no. 7, pp. 2229-2240, 2004.

[21] H. K. Lam, W.-K. Ling, H. H.-C. Iu, and S. S. H. Ling, "Synchronization of chaotic systems using time-delayed fuzzy state-feedback controller," IEEE Transactions on Circuits and Systems I, vol. 55, no. 3, pp. 893-903, 2008.

[22] H.-H. Chen, G.-J. Sheu, Y.-L. Lin, and C.-S. Chen, "Chaos synchronization between two different chaotic systems via nonlinear feedback control," Nonlinear Analysis. Theory, Methods \& Applications, vol. 70, no. 12, pp. 4393-4401, 2009.

[23] M. Liu, "Optimal exponential synchronization of general chaotic delayed neural networks: an LMI approach," Neural Networks, vol. 22, no. 7, pp. 949-957, 2009.

[24] Y.-Y. Hou, T.-L. Liao, and J.-J. Yan, " $H_{\infty}$ synchronization of chaotic systems using output feedback control design," Physica A, vol. 379, no. 1, pp. 81-89, 2007.

[25] D. Qi, M. Liu, M. Qiu, and S. Zhang, "Exponential $H_{\infty}$ synchronization of general discrete-time chaotic neural networks with or without time delays," IEEE Transactions on Neural Networks, vol. 21, no. 8, pp. 1358-1365, 2010.

[26] C. M. Lin, Y. F. Peng, and M. H. Lin, "CMAC-based adaptive backstepping synchronization of uncertain chaotic systems," Chaos, Solitons and Fractals, vol. 42, no. 2, pp. 981-988, 2009.

[27] C. K. Ahn, S.-T. Jung, S.-K. Kang, and S.-C. Joo, "Adaptive $H_{\infty}$ synchronization for uncertain chaotic systems with external disturbance," Communications in Nonlinear Science and Numerical Simulation, vol. 15, no. 8, pp. 2168-2177, 2010.

[28] C. F. Chuang, W. J. Wang, and Y. J. Chen, " $H_{\infty}$ synchronization of fuzzy model based chen chaotic systems," in Proceedings of the IEEE International Conference on Control Applications (CCA '10), pp. 1199-1204, Yokohama, Japan, September 2010.

[29] H. R. Karimi and P. Maass, "Delay-range-dependent exponential $H_{\infty}$ synchronization of a class of delayed neural networks," Chaos, Solitons and Fractals, vol. 41, no. 3, pp. 1125-1135, 2009.

[30] H. R. Karimi and H. Gao, "New delay-dependent exponential $H_{\infty}$ synchronization for uncertain neural networks with mixed time delays," IEEE Transactions on Systems, Man, and Cybernetics B, vol. 40, no. 1, pp. 173-185, 2010.

[31] B.-S. Chen, C.-H. Chiang, and S. K. Nguang, "Robust $H_{\infty}$ synchronization design of nonlinear coupled network via fuzzy interpolation method," IEEE Transactions on Circuits and Systems I, vol. 58, no. 2, pp. 349-362, 2011.

[32] S.-J. Wu, H.-H. Chiang, H.-T. Lin, and T.-T. Lee, "Neuralnetwork-based optimal fuzzy controller design for nonlinear systems," Fuzzy Sets and Systems, vol. 154, no. 2, pp. 182-207, 2005.

[33] J. T. Tsai, J. H. Chou, and T. K. Liu, "Tuning the structure and parameters of a neural network by using hybrid Taguchi-genetic algorithm," IEEE Transactions on Neural Networks, vol. 17, no. 1, pp. 69-80, 2006.

[34] A. Savran, "Multifeedback-layer neural network," IEEE Transactions on Neural Networks, vol. 18, no. 2, pp. 373-384, 2007.

[35] A. Alessandri, C. Cervellera, and M. Sanguineti, "Design of asymptotic estimators: an approach based on neural networks and nonlinear programming," IEEE Transactions on Neural Networks, vol. 18, no. 1, pp. 86-96, 2007.

[36] F. H. Hsiao, S. D. Xu, C. Y. Lin, and Z. R. Tsai, "Robustness design of fuzzy control for nonlinear multiple time-delay large-scale systems via neural-network-based approach," IEEE Transactions on Systems, Man, and Cybernetics B, vol. 38, no. 1, pp. 244-251, 2008.

[37] P. M. Patre, S. Bhasin, Z. D. Wilcox, and W. E. Dixon, "Composite adaptation for neural network-based controllers," IEEE Transactions on Automatic Control, vol. 55, no. 4, pp. 944950, 2010.

[38] H. O. Wang, K. Tanaka, and M. Griffin, "Parallel distributed compensation of nonlinear systems by Takagi-Sugeno fuzzy model," in Proceedings of the IEEE International Conference on Fuzzy Systems, pp. 531-538, March 1995.

[39] K. Tanaka, T. Hori, and H. O. Wang, "A multiple Lyapunov function approach to stabilization of fuzzy control systems," IEEE Transactions on Fuzzy Systems, vol. 11, no. 4, pp. 582-589, 2003.

[40] C. H. Sun and W. J. Wang, "An improved stability criterion for T-S fuzzy discrete systems via vertex expression," IEEE Transactions on Systems, Man, and Cybernetics B, vol. 36, no. 3, pp. 672-678, 2006.

[41] H. K. Lam, "Stability analysis of interval type-2 fuzzy-modelbased control systems IEEE Trans," IEEE Transactions on Systems, Man, and Cybernetics B, vol. 38, no. 3, pp. 617-628, 2008.

[42] K. Kiriakidis, "Fuzzy model-based control of complex plants," IEEE Transactions on Fuzzy Systems, vol. 6, no. 4, pp. 517-529, 1998.

[43] B. S. Chen, C. S. Tseng, and H. J. Uang, "Robustness design of nonlinear dynamic systems via fuzzy linear control," IEEE Transactions on Fuzzy Systems, vol. 7, no. 5, pp. 571-585, 1999.

[44] B. S. Chen, C. S. Tseng, and H. J. Uang, "Mixed $H_{2} / H_{\infty}$ fuzzy output feedback control design for nonlinear dynamic systems: an LMI approach," IEEE Transactions on Fuzzy Systems, vol. 8, no. 3, pp. 249-265, 2000.

[45] Y. Y. Cao and P. M. Frank, "Robust $H^{\infty}$ disturbance attenuation for a class of uncertain discrete-time fuzzy systems," IEEE Transactions on Fuzzy Systems, vol. 8, no. 4, pp. 406-415, 2000.

[46] Y. Y. Cao and Z. Lin, "Robust stability analysis and fuzzyscheduling control for nonlinear systems subject to actuator saturation," IEEE Transactions on Fuzzy Systems, vol. 11, no. 1, pp. 57-67, 2003.

[47] S. Limanond and J. Si, "Neural-network-based control design: an LMI approach," IEEE Transactions on Neural Networks, vol. 9, no. 6, pp. 1422-1429, 1998.

[48] S. Boyd, L. El Ghaoui, E. Feron, and V. Balakrishnan, Linear Matrix Inequalities in System and Control Theory, vol. 15 of SIAM Studies in Applied Mathematics, SIAM, Philadelphia, Pa, USA, 1994. 
[49] Y. J. Sun, "Exponential synchronization between two classes of chaotic systems," Chaos, Solitons and Fractals, vol. 39, no. 5, pp. 2363-2368, 2009.

[50] W. J. Wang and C. F. Cheng, "Stabilising controller and observer synthesis for uncertain large-scale systems by the Riccati equation approach," IEE Proceedings D, vol. 139, no. 1, pp. 72-78, 1992.

[51] P. Gahinet, A. Nemirovski, A. J. Laub, and M. Chilali, LMI Control Toolbox User's Guide, The MathWorks, 1995. 


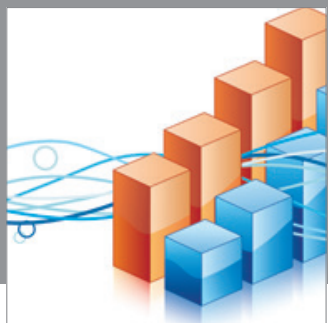

Advances in

Operations Research

mansans

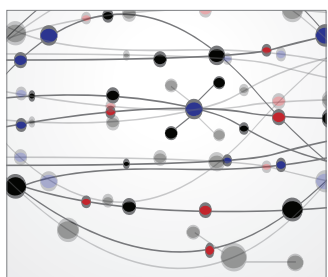

The Scientific World Journal
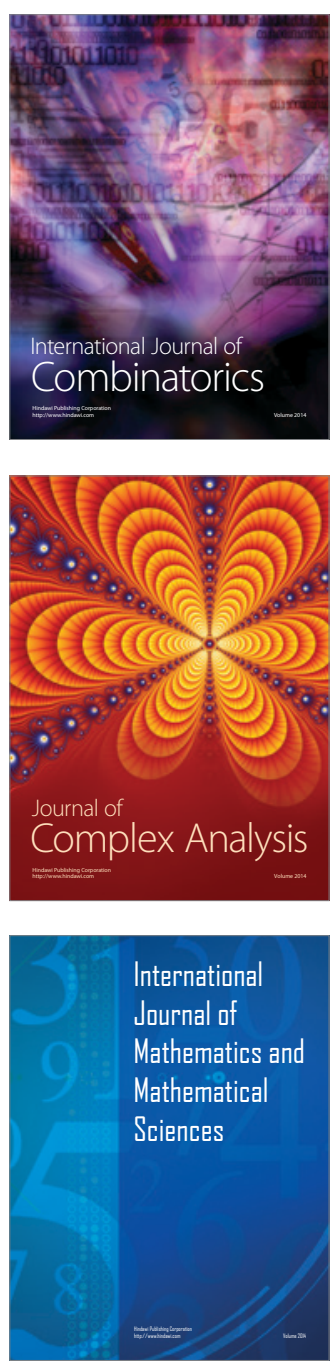
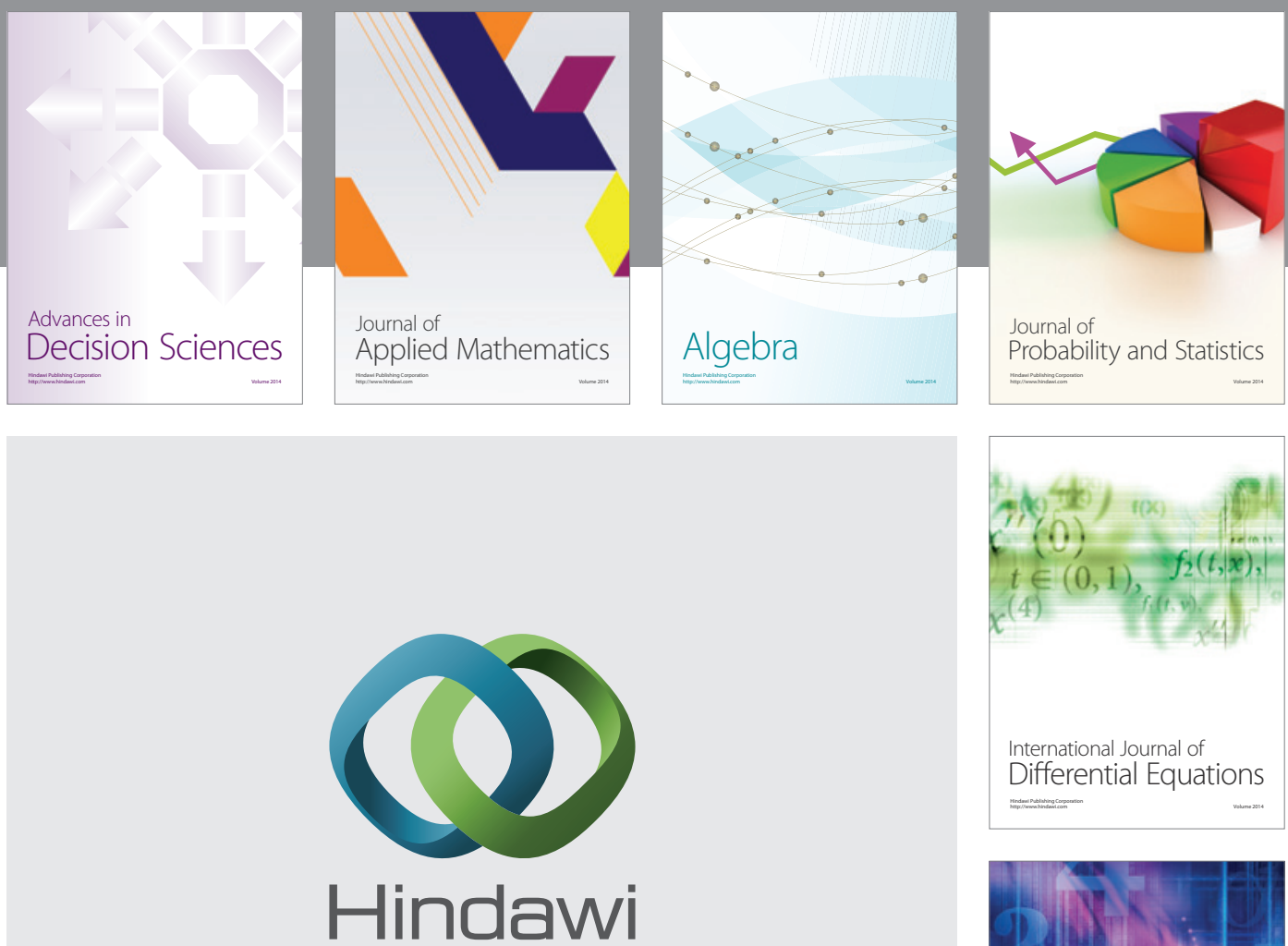

Submit your manuscripts at http://www.hindawi.com
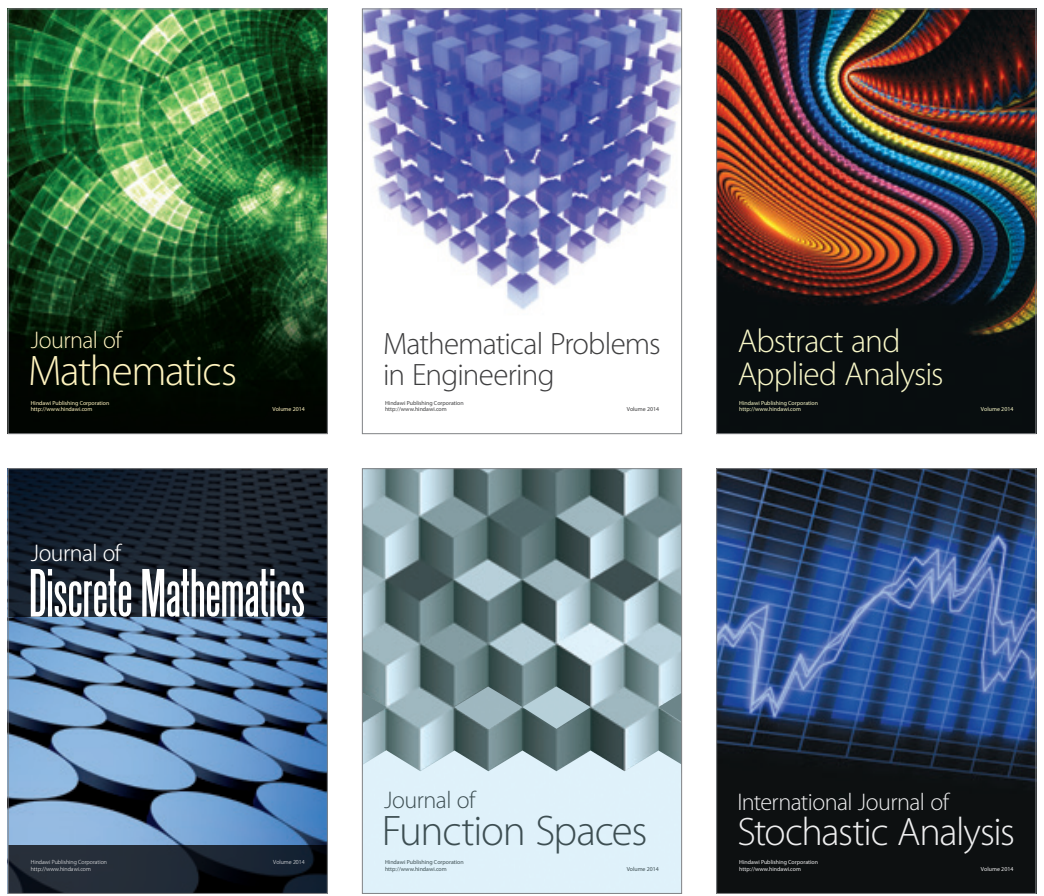

Journal of

Function Spaces

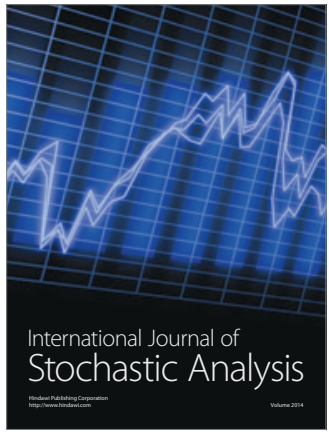

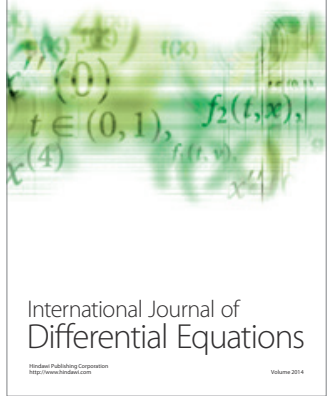
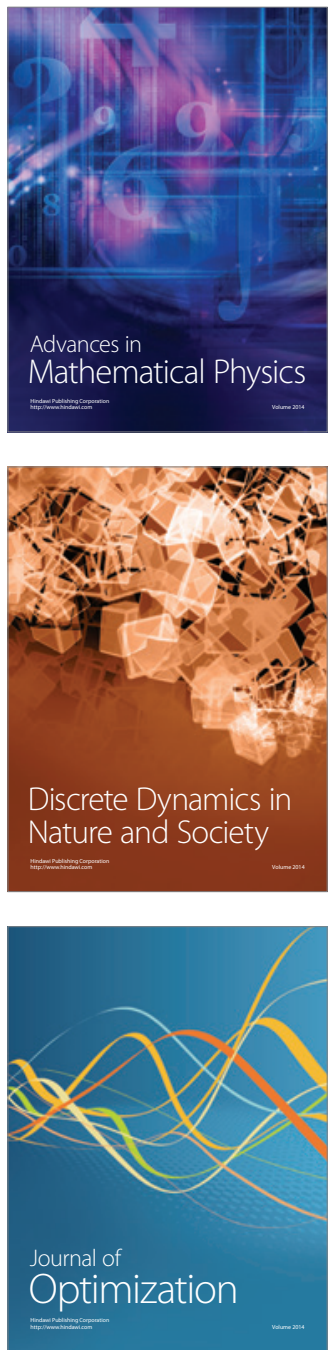